\title{
Theoretical Studies of Quantum Amplified Isomerizations for Imaging Systems Involving Hexamethyl Dewar Benzene and Related Systems
}

\author{
Joseph E. Norton, ${ }^{\dagger}$ Leif P. Olson, ${ }^{\ddagger}$ and K. N. Houk ${ }^{*}{ }^{\dagger}$ \\ Contribution from the Department of Chemistry and Biochemistry, University of \\ California, Los Angeles, California 90095-1569 and Research \& Development, Eastman \\ Kodak Company, Rochester, New York 14650-2109
}

\section{Supporting Information}

\section{Computational Details, Methodology, and Theory}

DFT. All calculations were carried out using the Gaussian 03 suite of programs. The hybrid density functional Becke3LYP ${ }^{1,2}$ with the $6-31 G(d)$ and $6-311+G(d, p)$ basis sets were used for all DFT calculations.

CASSCF. CASSCF calculations were carried out for Dewar benzene and hexamethyl Dewar benzene using the 6-31G(d) basis set with a six-electron six-orbital active space for neutral systems and a five-electron six-orbital active space for radical cation systems. Both active spaces comprise the $\pi$ and $\pi^{*}$ orbitals for benzene and the $\pi$ and $\pi^{*}$ orbitals and $\sigma$ and $\sigma *$ orbitals of the bridge carbons for Dewar benzene. Conical intersections and gradient difference and nonadiabatic derivative coupling vectors were calculated using the opt=Conical option.

Geometry Optimizations. All stationary structures were fully optimized and found to be ground state structures with zero imaginary frequencies. Transition structures were fully optimized and found to be first-order saddle points with s single vibrational frequency corresponding to the appropriate reaction coordinate. Potential energy surfaces for Dewar benzene and hexamethyl Dewar benzene were performed using the opt=Modredundant option to constrain the system along the flap dihedral and transannular $\mathrm{C}-\mathrm{C}$ bond distance reaction coordinates. Each surface comprises a total of 442 optimized structures.

Ionization Potentials and Electron Affinities. Vertical and adiabatic ionization potentials and electron affinities were calculated using relative electronic energies. The vertical ionization potential is the difference in energy between the optimized neutral reactant and a corresponding single-point energy calculation of the radical cation. The vertical electron affinity is the energy difference between the optimized radical cation product and a corresponding single-point energy calculation of the neutral state.

\footnotetext{
${ }^{\dagger}$ University of California, Los Angeles.

${ }^{\ddagger}$ Eastman Kodak Company.

* Corresponding author. Mailing address: University of California, Los Angeles, Department of Chemistry and Biochemistry, 607 Charles E. Young Drive East, Box 951569, Los Angeles, CA 90095-1569.

Telephone: 310-206-0515. Fax: 310-206-1843. E-mail: houk@chem.ucla.edu.
} 
Adiabatic ionization potential and electron affinity are the energy differences between the optimized neutral state and the optimized radical cation state.

Electron-Transfer Theory. Electron transfer rates were calculated using the following equations, based on Marcus thoery ${ }^{3,4}$ and other well-known electron-transfer theories: ${ }^{5-10}$

$$
\begin{gathered}
k=\left(\frac{4 \pi^{3}}{h^{2} \lambda_{s} k_{B} T}\right)^{1 / 2}|V|^{2} \sum_{w=0}^{\infty}\left(\frac{e^{-S} S^{w}}{w !}\right) \exp \left\{-\frac{\left(\lambda_{s}+\Delta G+w h v\right)^{2}}{4 \lambda_{s} k_{B} T}\right\} \\
S=\frac{\lambda_{v}}{h v}
\end{gathered}
$$

where $V, \lambda_{s}, \lambda_{v}, v$, and $\Delta G$ are the electron coupling matrix element, solvent reorganization energy, vibration reorganization energy, single average frequency, and free energy change for the electron-transfer process, respectively. Additionally, $h, k_{\mathrm{B}}$, and $T$ are Planck's constant, Boltzmann's constant, and temperature, respectively. Here, $\Delta G$ for forward electron transfer $\left(\Delta G_{\mathrm{f}}\right)$ was calculated according to the Rehm-Weller eq $3^{11}$ and $\Delta G$ for backward electron transfer $\left(\Delta G_{\mathrm{b}}\right)$ was calculated according to eq 4 of Marcus theory.

$$
\begin{aligned}
\Delta G_{f} & =E_{1 / 2}^{o x}-E_{1 / 2}^{r e d}-\frac{e^{2}}{\varepsilon r}-E\left(S_{1}\right) \\
\Delta G_{b} & =E_{1 / 2}^{r e d}-E_{1 / 2}^{o x}+\frac{e^{2}}{\varepsilon r}
\end{aligned}
$$

$E_{1 / 2}^{o x}, E_{1 / 2}^{r e d}, E\left(S_{1}\right)$, and $\frac{e^{2}}{\varepsilon r}$ are the oxidation potential of the electron donor, the reduction potential of the electron acceptor, the excitation energy of the fluorescent state, and the Coulomb attraction energy for oppositely monocharged radical ion pairs, respectively. Also, $e$ is the elementary charge of an electron, $\varepsilon$ is the dielectric constant of the surrounding medium, and $r$ is the separation distance between donor and acceptor, which can be estimated as the sum of the radii of the donor and acceptor. Radii were determined using the Monte Carlo integration method implemented in Gaussian 03. The Volume=Tight keyword was used to obtain a radius $(0.5 \AA$ larger than the radius corresponding to the computed volume), which is usually used in conjunction with the Onsager solvent reaction field model.

Reorganization energy is defined as "the energy associated with the structural adjustments needed for the reactants to assume the configuration required for the transfer of one electron.” Here, these values were determined as the difference in energy between the optimized neutral geometry and a single-point energy calculation of the neutral state at the optimized radical cation geometry. This energy difference is the vibrational reorganization energy of the solute. The total vibrational reorganization energy, $\lambda_{v}$, is the sum of the donor $\left(\lambda_{\mathrm{D}}\right)$ and acceptor $\left(\lambda_{\mathrm{A}}\right)$ vibrational reorganization energies.

Solvent reorganization energies were calculated using eq 5 from Marcus theory ${ }^{3,4}$ :

$$
\lambda_{s}=\frac{e^{2}}{2}\left(\frac{1}{r_{A}}+\frac{1}{r_{D}}-\frac{2}{r}\right)\left(\frac{1}{n^{2}}-\frac{1}{\varepsilon}\right)
$$


Here, $r_{\mathrm{A}}$ and $r_{\mathrm{D}}$ are the radii of the acceptor and donor molecules, respectively, and $\mathrm{r}$ is the separation distance between the two, which can be estimated as the sum of $r_{\mathrm{A}}$ and $r_{\mathrm{D}}$. $n$ is the refractive index of the surrounding medium, and $\varepsilon$ is its dielectric constant.

Redox Potentials. Values for $\Delta G$ were obtained by calculating the corresponding redox potentials using a method similar to that used by Friesner et al. ${ }^{12}$ and Dutton et al. ${ }^{13}$ Standard one-electron redox potentials, $E^{0}$, are related to the total electron attachment energy in solution, $\Delta G_{r x n, \text { solv }}$, by

$$
\Delta G_{r \times n, \text { solv }}=-F E^{0}
$$

where $F$ is the Faraday constant. $\Delta G_{r x n, \text { solv }}$ can be calculated computationally according to the energy components.

$$
\Delta G_{r x n, \text { solv }}=\Delta G_{\text {gas }}+\Delta G_{\text {solv }}
$$

From this equation, $\Delta G_{g a s}$ is the sum of electronic and thermal free energies at $298 \mathrm{~K}$ and $\Delta G_{\text {solv }}$ is the calculated free energy of solvation. Diethyl ether was used as the solvent for calculating $\Delta G_{\text {solv }}$ for Dewar benzene and hexamethyl Dewar benzene.

For all redox potentials, geometry optimizations were performed at the B3LYP/6$311+G(d, p)$ level of theory. Free energies of solvation were calculated using the conductor-like polarizable continuum model (CPCM) with the United Atom Topological Model cavity model optimized for the HF/6-31G(d) level of theory (UAHF). Solvation energies were obtained from B3LYP/6-311+G(d,p) single-point calculations of B3LYP/6-311+G(d,p) gas-phase geometry optimized structures with diethyl ether $(\varepsilon=$ 4.335, $n=1.3542$ ) as the solvent. The absolute reduction potential of the normal hydrogen electrode (NHE) has been determined experimentally to be $4.43 \mathrm{~V}^{14}$ Given that the reduction potential of the electron acceptor used here, $E_{\frac{1}{2}}^{r e d}$, is obtained from experimental values vs SCE, ${ }^{15}$ we reference our computed absolute potentials to the commonly used standard calomel electrode (SCE). The standard potential of SCE is $+0.2412 \mathrm{~V}$ vs NHE. ${ }^{16}$ To adjust the computed absolute potentials with experimental potentials vs SCE, $4.67 \mathrm{~V}$ was subtracted from the computed absolute values.

Frequency-Dependent Index of Refraction. Indices of refraction were calculated using the Lorentz-Lorenz equation. ${ }^{17-21}$

$$
n(\omega)=\sqrt{\frac{3+8 \pi N \alpha(\omega)}{3-4 \pi N \alpha(\omega)}}
$$

This form of the Lorentz-Lorenz expression requires the frequency-dependent polarizability, $\alpha(\omega)$, and the number of molecules per unit volume, $N$. Polarizability is calculated quantum mechanically with Gaussian 03 as a tensor quantity in terms of its individual tensors. The index of refraction of interest for amorphous materials such as organic liquids is the average isotropic polarizability. Values of $N$ can be determined from the density of experimentally known systems or can be approximated computationally. The GRASP program was used to determine $N$ when experimental data were lacking. 
The GRASP program was used to generate a relationship between calculated molecular surface volumes ( $V_{\text {GRASP }}$ ) and experimentally known condensed-phase molar volumes ( $V_{\text {exp }}$ ) from which values of $N$ could be determined. A test set comprising a number of small aromatic systems (Table 1 ) was used to generate the relationship, $V_{\text {GRASP }}=1.8901$ $V_{\text {exp }}-6.5937$ with $\mathrm{R}^{2}=0.9789$ and $\mathrm{RMSD}=5.87 \%$. Figure 1 shows the correlation between experimental and calculated molecular volumes and the relationship generated from linear regression analysis. 
Table 1. Data for Correlation of GRASP Molecular Surface Volumes with Experimental Molecular Volumes Determined from Density. GRASP Volumes were Calculated Using the Default Probe Radius of $1.40 \AA$

\begin{tabular}{|c|c|c|c|c|c|c|c|}
\hline & $T(K)$ & $\begin{array}{l}\text { Density } \\
(\mathrm{g} / \mathrm{mL})^{a}\end{array}$ & MW & $\begin{array}{c}\text { Molecular } \\
\text { Volume } \\
\left(\AA^{3} / \text { molecule }\right)\end{array}$ & $\begin{array}{c}\text { GRASP } \\
\text { Molecular } \\
\text { Surface } \\
\text { Volume }\left(\AA^{3}\right)\end{array}$ & $\begin{array}{c}\text { Standard } \\
\text { Error }\end{array}$ & $\begin{array}{l}\text { Standard } \\
\text { Error (\%) }\end{array}$ \\
\hline Benzaldehyde & 298 & 1.045 & 106.12 & 168.69 & 100.51 & 215.86 & 75.86 \\
\hline Aniline & 298 & 1.022 & 93.13 & 151.37 & 90.07 & 150.72 & 65.78 \\
\hline Phenol & 298 & 1.071 & 94.11 & 145.97 & 87.70 & 174.31 & 81.81 \\
\hline Ethylbenzene & 298 & 0.867 & 106.17 & 203.42 & 109.34 & 11.20 & 2.71 \\
\hline$m$-Xylene & 298 & 0.868 & 106.17 & 203.18 & 108.95 & 14.82 & 3.59 \\
\hline o-Xylene & 298 & 0.879 & 106.17 & 200.64 & 109.12 & 0.97 & 0.24 \\
\hline Toluene & 298 & 0.865 & 92.14 & 176.94 & 93.04 & 59.02 & 18.85 \\
\hline Benzene & 298 & 0.874 & 78.11 & 148.46 & 77.01 & 90.13 & 40.89 \\
\hline Hexamethyl Dewar Benzene & 298 & 0.803 & 162.27 & 335.68 & 179.06 & 14.69 & 1.30 \\
\hline Pyrrole & 298 & 0.967 & 67.09 & 115.25 & 64.48 & 0.00 & 0.00 \\
\hline Furan & 298 & 0.936 & 68.07 & 120.80 & 62.88 & 73.08 & 50.08 \\
\hline \multirow[t]{2}{*}{ Thiophene } & 298 & 1.051 & 84.14 & 132.99 & 72.56 & 5.92 & 3.35 \\
\hline & & & & & & $\begin{array}{c}\text { RMSE } \\
9.00\end{array}$ & $\begin{array}{c}\text { RMSE (\%) } \\
5.87\end{array}$ \\
\hline
\end{tabular}

${ }^{a}$ Molecular volumes obtained from experimental densities from the Sigma-Aldrich database.

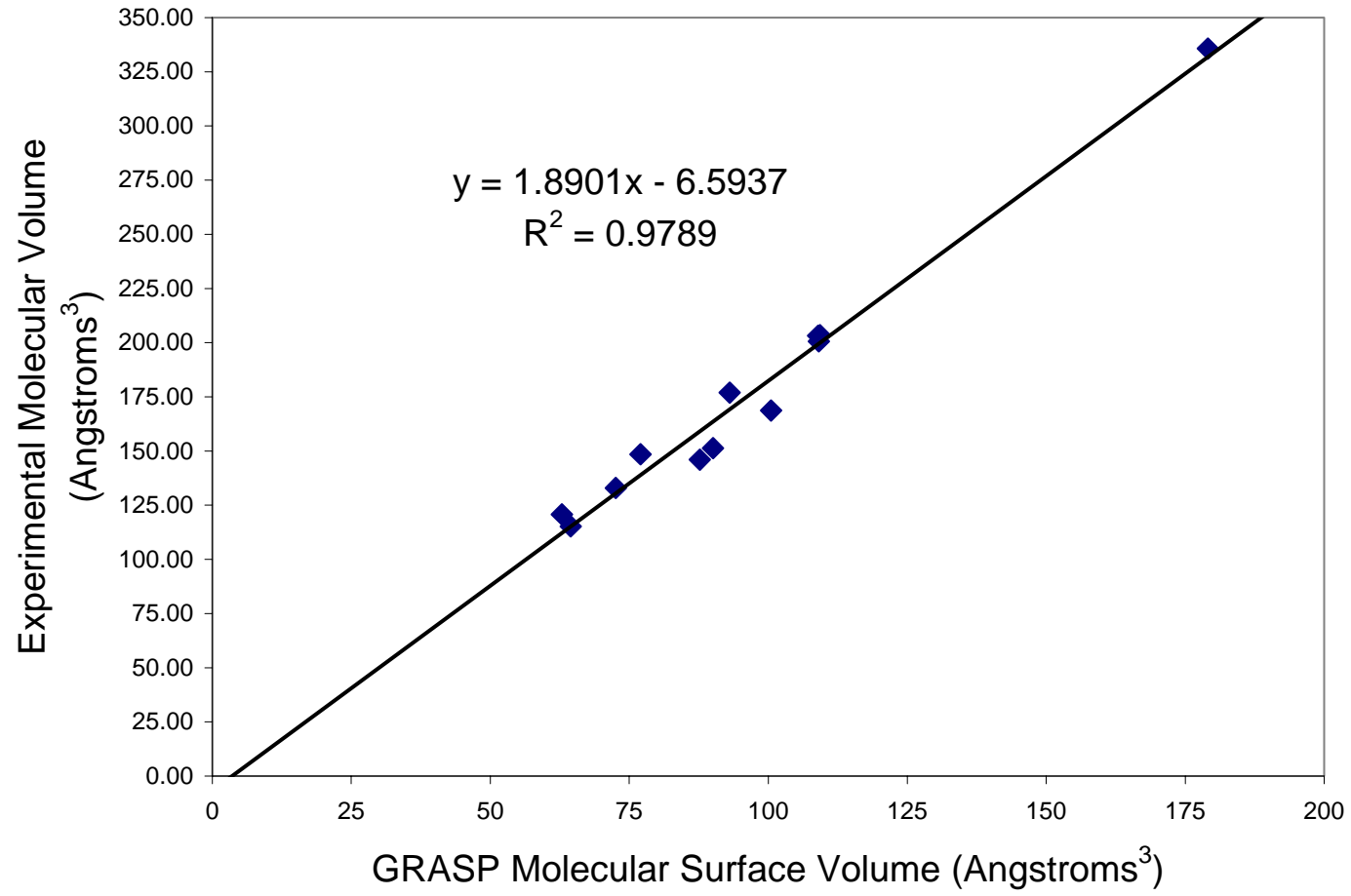

Figure 1. Correlation of experimental molecular volumes with volumes of molecular surfaces calculated using the GRASP program. 


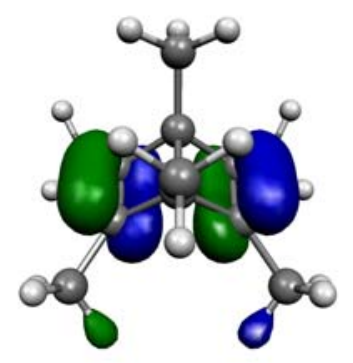

${ }^{2} \mathrm{~B}_{2}$

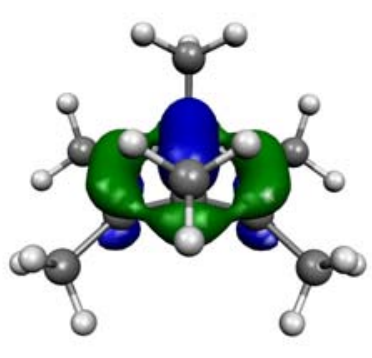

${ }^{2} \mathrm{~A}_{1}$

Figure 2. B3LYP/6-31G(d) SOMOs of the radical cation states of 1 . The ${ }^{2} \mathrm{~B}_{2}$ radical cation state has the spin localized on the $\pi$ orbitals while the ${ }^{2} \mathrm{~A}_{1}$ radical cation has the spin localized on the elongated transannular $\mathrm{C}-\mathrm{C} \sigma$ orbital. 


\section{References}

(1) Becke, A. D. J. Chem. Phys. 1993, 98, 5648-5652.

(2) Lee, C.; Yang, W.; Parr, R. G. Phys. Rev. B: Condens. Matter Mater. Phys. 1988, 37, 785-789.

(3) Marcus, R. A. J. Chem. Phys. 1956, 24, 966-978.

(4) Marcus, R. A.; Eyring, H. Ann. Rev. Phys. Chem. 1964, 15, 155-196.

(5) Van Duyne, R. P.; Fischer, S. F. Chem. Phys. 1974, 5, 183-97.

(6) Ulstrup, J.; Jortner, J. J. Chem. Phys. 1975, 63, 4358-68.

(7) Siders, P.; Marcus, R. A. J. Am. Chem. Soc. 1981, 103, 741-747.

(8) Siders, P.; Marcus, R. A. J. Am. Chem. Soc. 1981, 103, 748-752.

(9) Miller, J. R.; Beitz, J. V.; Huddleston, R. K. J. Am. Chem. Soc. 1984, 106, 50575068.

(10) Wolfram Research, Inc., Mathematica, Version 4.0, Champaign, IL, 1999.

(11) Rehm, D.; Weller, A. Isr. J. Chem. 1970, 8, 259-71.

(12) Baik, M.-H.; Friesner, R. A. J. Phys. Chem. A 2002, 106, 7407-7412.

(13) Dutton, A. S.; Fukuto, J. M.; Houk, K. N. Inorg. Chem. 2005, 44, 4024-4028.

(14) Reiss, H.; Heller, A. J. Phys. Chem. 1985, 89, 4207-4213.

(15) Kiau, S.; Liu, G.; Shukla, D.; Dinnocenzo, J. P.; Young, R. H.; Farid, S. J. Phys. Chem. A 2003, 107, 3625-3632.

(16) Bard, A. J.; Faulkner, L. R. Electrochemical Methods: Fundamentals and Applications; 2nd ed.; John Wiley \& Sons: New York, 2000.

(17) Lorentz, H. A. The Theory of Electrons; Dover: New York, 1952.

(18) Böttcher, C. J. F. Theory of Electric Polarization; 2nd ed.; Elsevier: Amsterdam, 1973.

(19) Nussbaum, A.; Phillips, R. A. Contemporary Optics for Scientists and Engineers; Prentice-Hall: Englewood Cliffs, N.J., 1976.

(20) Sylvester-Hvid, K. O.; Astrand, P.-O.; Ratner, M. A.; Mikkelsen, K. V. J. Phys. Chem. A 1999, 103, 1818-1821.

(21) Sylvester-Hvid, K. O.; Mikkelsen, K. V.; Ratner, M. A. J. Phys. Chem. A 1999, 103, 8447-8457. 


\section{Software Citations}

\section{Gaussian 03}

Gaussian 03, Revision C.02, Frisch, M. J.; Trucks, G. W.; Schlegel, H. B.; Scuseria, G. E.; Robb, M. A.; Cheeseman, J. R.; Montgomery, J., J. A.; Vreven, T.; Kudin, K. N.; Burant, J. C.; Millam, J. M.; Iyengar, S. S.; Tomasi, J.; Barone, V.; Mennucci, B.; Cossi, M.; Scalmani, G.; Rega, N.; Petersson, G. A.; Nakatsuji, H.; Hada, M.; Ehara, M.; Toyota, K.; Fukuda, R.; Hasegawa, J.; Ishida, M.; Nakajima, T.; Honda, Y.; Kitao, O.; Nakai, H.; Klene, M.; Li, X.; Knox, J. E.; Hratchian, H. P.; Cross, J. B.; Adamo, C.; Jaramillo, J.; Gomperts, R.; Stratmann, R. E.; Yazyev, O.; Austin, A. J.; Cammi, R.; Pomelli, C.; Ochterski, J. W.; Ayala, P. Y.; Morokuma, K.; Voth, G. A.; Salvador, P.; Dannenberg, J. J.; Zakrzewski, V. G.; Dapprich, S.; Daniels, A. D.; Strain, M. C.; Farkas, O.; Malick, D. K.; Rabuck, A. D.; Raghavachari, K.; Foresman, J. B.; Ortiz, J. V.; Cui, Q.; Baboul, A. G.; Clifford, S.; Cioslowski, J.; Stefanov, B. B.; Liu, G.; Liashenko, A.; Piskorz, P.; Komaromi, I.; Martin, R. L.; Fox, D. J.; Keith, T.; Al-Laham, M. A.; Peng, C. Y.; Nanayakkara, A.; Challacombe, M.; Gill, P. M. W.; Johnson, B.; Chen, W.; Wong, M. W.; Gonzalez, C.; and Pople, J. A., Gaussian, Inc.,Wallingford CT, 2004.

\section{GRASP}

Nicholls, A.; Sharp, K.; Honig, B. PROTEINS, Structure, Function, and Genetics 1991, $11,281 \mathrm{ff}$. 


\section{Geometry Summaries}

Cartesian coordinates and distances are reported in angstroms. Electronic energies are reported in Hartrees. Charge/multiplicity is specified for each structure.

DB (Dewar Benzene) 0/1

RB3LYP/6-311+G(d,p) optimized structure:

$\mathrm{C}$
$\mathrm{C}$
$\mathrm{C}$
$\mathrm{C}$
$\mathrm{C}$
$\mathrm{C}$
$\mathrm{H}$
$\mathrm{H}$
$\mathrm{H}$
$\mathrm{H}$
$\mathrm{H}$
$\mathrm{H}$

$\begin{array}{rrr}0.070126 & 0.081532 & -0.095039 \\ -0.256884 & -0.238844 & 1.364921 \\ 0.837795 & 0.023242 & 2.401396 \\ 1.470509 & -1.134950 & 2.179983 \\ 0.488667 & -1.603191 & 1.103909 \\ 0.703115 & -1.076446 & -0.316678 \\ -0.106354 & 0.964774 & -0.697341 \\ -1.298721 & -0.216307 & 1.685993 \\ 1.041732 & 0.877600 & 3.035818 \\ 2.381793 & -1.575394 & 2.566746 \\ -0.018809 & -2.559053 & 1.237313 \\ 1.234408 & -1.487730 & -1.166716 \\ & & \end{array}$

$\pi$ RC (Dewar Benzene) $1 / 2$

UB3LYP/6-311+G(d,p) optimized structure:

$\begin{array}{lr}\text { C } & -1.191368 \\ \text { C } & -0.000127 \\ \text { C } & -1.191598 \\ \text { C } & 0.000220 \\ \text { C } & 1.191346 \\ \mathrm{C} & 1.191576 \\ \mathrm{H} & -1.782351 \\ \mathrm{H} & -1.782678 \\ \mathrm{H} & 0.000579 \\ \mathrm{H} & -0.000374 \\ \mathrm{H} & 1.782551 \\ \mathrm{H} & 1.782223\end{array}$

$$
\begin{array}{r}
-0.689878 \\
-0.790221 \\
0.690300 \\
0.790215 \\
0.689874 \\
-0.690290 \\
-1.434917 \\
1.435614 \\
1.427213 \\
-1.427226 \\
-1.435609 \\
1.434927
\end{array}
$$$$
\begin{array}{r}
-0.306400 \\
0.631755 \\
-0.305738 \\
0.631762 \\
-0.306567 \\
-0.305917 \\
-0.826727 \\
-0.825552 \\
1.510942 \\
1.510929 \\
-0.825842 \\
-0.826993
\end{array}
$$

$E(U B+H F-L Y P)=-231.857155210$

\begin{tabular}{|c|c|c|c|}
\hline C & $-\odot .007711$ & $\odot .00 \odot \odot 4 \odot$ & $-\odot .04076 \odot$ \\
\hline C & 0.011880 & $\odot .00 \odot 492$ & 1.388849 \\
\hline C & 1.238807 & -0.000069 & 2.120479 \\
\hline C & 2.423745 & -0.001018 & 1.435986 \\
\hline C & 2.404155 & $-\odot .001475$ & $\odot .0 \odot 6386$ \\
\hline C & 1.177221 & $-\odot . \odot \odot \odot 9 \odot 9$ & -0.725250 \\
\hline $\mathrm{H}$ & -0.958357 & $\odot .000470$ & -0.55953 \\
\hline $\mathrm{H}$ & -0.928197 & $\odot .001288$ & 1.93088 \\
\hline $\mathrm{H}$ & 1.213255 & $\odot . \odot \odot \odot 276$ & 3.20312 \\
\hline $\mathrm{H}$ & 3.374384 & $-\odot .001447$ & 1.95477 \\
\hline $\mathrm{H}$ & 3.344224 & $-0.0 \odot 2308$ & -0.53566 \\
\hline $\mathrm{H}$ & 1.202790 & -0.001251 & -1.80789 \\
\hline \multicolumn{2}{|c|}{$E(U B+H F-L Y P)=$} & -231.975695096 & \\
\hline
\end{tabular}

$\pi-\sigma$ TS (Dewar Benzene) $1 / 2$ $U B 3 L Y P / 6-311+G(d, p)$ optimized structure:

$$
\begin{array}{lrrr}
\text { C } & -1.325543 & -0.664543 & -0.272515 \\
\mathrm{C} & 0.002585 & -0.850703 & 0.472073 \\
\mathrm{C} & -1.325782 & 0.664177 & -0.273779 \\
\mathrm{C} & 0.001645 & 0.852234 & 0.470698 \\
\mathrm{C} & 1.222009 & 0.697468 & -0.355438 \\
\mathrm{C} & 1.221335 & -0.698317 & -0.356753 \\
\mathrm{H} & -1.943636 & -1.434927 & -0.714464 \\
\mathrm{H} & -1.945200 & 1.433016 & -0.716482 \\
\mathrm{H} & 0.045446 & 1.446636 & 1.381811 \\
\mathrm{H} & 0.047354 & -1.443007 & 1.384483 \\
\mathrm{H} & 1.998051 & -1.404632 & -0.635176 \\
\mathrm{H} & 2.001735 & 1.402602 & -0.628807 \\
& & &
\end{array}
$$

$\sigma$ RC (Dewar Benzene) $1 / 2$

UB3LYP/6-311+G(d,p) optimized structure:

$\begin{array}{lrrr}\text { C } & 0.969712 & 0.000000 & 0.314373 \\ \text { C } & 0.669056 & 1.307806 & -0.339475 \\ \text { C } & 0.669056 & -1.307806 & -0.339475 \\ \text { C } & -0.669057 & -1.307808 & -0.339474 \\ \text { C } & -0.669057 & 1.307808 & -0.339474 \\ \text { C } & -0.969721 & 0.000000 & 0.314367 \\ \text { H } & -1.391247 & -2.081215 & -0.572989 \\ \text { H } & 1.391249 & -2.081209 & -0.572994 \\ \text { H } & 1.570835 & 0.000000 & 1.224861 \\ \text { H } & -1.570828 & 0.000000 & 1.224866 \\ \text { H } & -1.391247 & 2.081215 & -0.572990 \\ \text { H } & 1.391250 & 2.081209 & -0.572994 \\ \text { E (UB+HF-LYP })= & & \end{array}$

CTS (Dewar Benzene) 1/2

UB3LYP/6-311+G(d,p) optimized structure:

$\begin{array}{rrrr}\text { C } & -1.289424 & -0.718820 & -0.218615 \\ \text { C } & 0.000781 & -0.990363 & 0.455082 \\ \text { C } & -1.298838 & 0.622898 & -0.206539 \\ \text { C } & -0.000650 & 1.129341 & 0.287576 \\ \text { C } & 1.298363 & 0.624640 & -0.206140 \\ \text { C } & 1.290792 & -0.717101 & -0.218261 \\ \text { H } & -2.043384 & -1.443645 & -0.498308 \\ \text { H } & -2.135812 & 1.284385 & -0.404920 \\ \text { H } & -0.001319 & 2.000347 & 0.940650 \\ \text { H } & 0.000865 & -1.323702 & 1.495267 \\ \text { H } & 2.045854 & -1.440865 & -0.497725 \\ \text { H } & 2.134535 & 1.287251 & -0.404171 \\ & & & \\ \text { E (UB+HF-LYP })= & -231.852740329 & \end{array}$

BRC (Benzene) $1 / 2$

UB3LYP/6-311+G(d,p) optimized structure:

$\begin{array}{llll}\text { C } & 0.000463 & 0.000053 & 0.000321 \\ \text { C } & 0.000397 & 0.000476 & 1.394792\end{array}$




$$
\begin{aligned}
& \mathrm{C} \\
& \mathrm{C} \\
& \mathrm{C} \\
& \mathrm{C} \\
& \mathrm{H} \\
& \mathrm{H} \\
& \mathrm{H} \\
& \mathrm{H} \\
& \mathrm{H} \\
& \mathrm{H}
\end{aligned}
$$

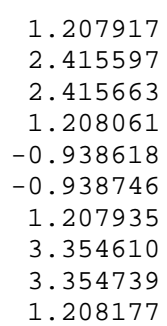

$-0.0000031$

$-0.001048$

$-0.001507$

$-0.000922$

$\odot .000487$

$\odot .001268$

0.000315

$-0.001466$

$-0.002280$

$-0.001256$
2.092083

1. 394861

0.000485

$-0.696853$

$-0.541837$

1.936850

3.176424

1.937139

$-0.541689$

$-1.781194$
$E(R B+H F-L Y P)=-232.311245820$

DB (Dewar Benzene) 0/1

CAS(6,6)/6-31G(d) optimized structure:

$\begin{array}{lrrr}\text { C } & 0.064774 & 0.079636 & -0.082548 \\ \text { C } & -0.271249 & -0.232546 & 1.369534 \\ \text { C } & 0.826218 & 0.021712 & 2.394148 \\ \text { C } & 1.461760 & -1.141469 & 2.171577 \\ \text { C } & 0.486540 & -1.619381 & 1.104121 \\ \text { C } & 0.700385 & -1.083504 & -0.305140 \\ \text { H } & -0.085608 & 0.962810 & -0.675936 \\ \text { H } & -1.304536 & -0.194972 & 1.688090 \\ \text { H } & 1.046594 & 0.876687 & 3.006662 \\ \text { H } & 2.379280 & -1.562403 & 2.539941 \\ \text { H } & -0.003984 & -2.575135 & 1.232553 \\ \text { H } & 1.247204 & -1.476201 & -1.142695 \\ & & & \\ \text { E= } & -230.6420927246 & \end{array}$

$\pi$ RC (Dewar Benzene) 1/2

CAS(5,6)/6-31G(d) optimized structure:

$\begin{array}{rrrr}\text { C } & -1.212410 & -0.702748 & -\odot .302312 \\ \text { C } & 0.001321 & -0.809603 & 0.575608 \\ \text { C } & -1.212325 & 0.703067 & -0.302175 \\ \text { C } & 0.001523 & 0.809596 & 0.575618 \\ \text { C } & 1.249560 & 0.673697 & -0.295262 \\ \text { C } & 1.249495 & -0.674008 & -0.295125 \\ \text { H } & -1.865243 & -1.419027 & -0.767547 \\ \text { H } & -1.865048 & 1.419519 & -0.767298 \\ \text { H } & -0.015784 & 1.441323 & 1.448692 \\ \text { H } & -0.016273 & -1.441350 & 1.448662 \\ \text { H } & 1.842516 & -1.422808 & -0.781471 \\ \text { H } & 1.842668 & 1.422343 & -0.781738 \\ & & & \\ \text { E= } & -230.3319789959 & \end{array}$

$\pi-\sigma$ TS (Dewar Benzene) $1 / 2$

CAS(5,6)/6-31G(d) optimized structure:

$\begin{array}{rrrr}\text { C } & -1.230789 & -0.694551 & -0.346166 \\ \text { C } & 0.001358 & -0.886959 & 0.444737 \\ \text { C } & -1.230645 & 0.694833 & -0.346120 \\ \text { C } & 0.001556 & 0.886974 & 0.444732 \\ \text { C } & 1.325630 & 0.666577 & -0.268483 \\ \text { C } & 1.325493 & -0.666889 & -0.268454 \\ \text { H } & -2.018838 & -1.381993 & -0.596730 \\ \text { H } & -2.018618 & 1.382425 & -0.596515 \\ \text { H } & -0.049051 & 1.509861 & 1.323894 \\ \text { H } & -0.049450 & -1.509799 & 1.323920 \\ \text { H } & 1.971515 & -1.416966 & -0.679563 \\ \text { H } & 1.971839 & 1.416490 & -0.679601 \\ & & & \\ \text { E= } & -230.3274378667 & \end{array}$

$\sigma$ RC (Dewar Benzene) 1/2

CAS(5,6)/6-31G(d) optimized structure:

$\begin{array}{rrrr}\text { C } & 1.039067 & 0.000000 & 0.323576 \\ \text { C } & 0.670077 & 1.290880 & -0.321337 \\ \text { C } & 0.670077 & -1.290883 & -0.321334 \\ \text { C } & -0.670061 & -1.290888 & -0.321309 \\ \text { C } & -0.670061 & 1.290884 & -0.321314 \\ \text { C } & -1.039088 & 0.00000 \odot & 0.323574 \\ \text { H } & -1.366199 & -2.064085 & -0.584331 \\ \text { H } & 1.366230 & -2.064052 & -0.584397 \\ \text { H } & 1.664224 & 0.000003 & 1.202117 \\ \text { H } & -1.664297 & 0.000002 & 1.202076 \\ \text { H } & -1.366197 & 2.064087 & -0.584323 \\ \text { H } & 1.366229 & 2.064052 & -0.584397 \\ \text { E } & -230.3412264340 & \end{array}$

CTS (Dewar Benzene) 1/2

CAS(5,6)/6-31G(d) optimized structure:

$\begin{array}{rrrr}\text { C } & -1.288549 & -0.682954 & -0.302602 \\ \text { C } & 0.001796 & -1.045593 & 0.342922 \\ \text { C } & -1.292160 & 0.657259 & -0.274858 \\ \text { C } & -0.000571 & 1.054358 & 0.350922 \\ \text { C } & 1.289870 & 0.660241 & -0.279110 \\ \text { C } & 1.289256 & -0.679959 & -0.306716 \\ \text { H } & -2.052249 & -1.382032 & -0.584364 \\ \text { H } & -2.082649 & 1.343145 & -0.513149 \\ \text { H } & -0.000006 & 1.736138 & 1.185469 \\ \text { H } & 0.003908 & -1.631266 & 1.248743 \\ \text { H } & 2.053502 & -1.377314 & -0.591259 \\ \text { H } & 2.077851 & 1.347978 & -0.520346 \\ & & \end{array}$

BRC (Benzene) 1/2

CAS(5,6)/6-31G(d) optimized structure:

$\begin{array}{rrrr}\text { C } & 0.011127 & 0.000047 & 0.006952 \\ \text { C } & -0.008026 & 0.000524 & 1.432491 \\ \text { C } & 1.178668 & -0.000016 & 2.117380 \\ \text { C } & 2.404906 & -0.001051 & 1.388276 \\ \text { C } & 2.424058 & -0.001504 & -0.037259 \\ \text { C } & 1.237363 & -0.000958 & -0.722149 \\ \text { H } & -0.918962 & 0.000477 & -0.530650 \\ \text { H } & -0.949511 & 0.001286 & 1.947491 \\ \text { H } & 1.203493 & 0.000307 & 3.190190 \\ \text { H } & 3.334995 & -0.001465 & 1.925879 \\ \text { H } & 3.365541 & -0.002268 & -0.552262 \\ \text { H } & 1.212542 & -0.001289 & -1.794959 \\ & & \end{array}$

B (Benzene) 0/1

CAS(6,6)/6-31G(d) optimized structure:

$\begin{array}{rrrr}\text { C } & -0.001267 & 0.000065 & -0.000689 \\ \text { C } & -0.001337 & 0.000552 & 1.395802 \\ \text { C } & 1.207904 & -0.000052 & 2.094037 \\ \text { C } & 2.417338 & -0.001027 & 1.395849 \\ \text { C } & 2.417408 & -0.001465 & -0.000503 \\ \text { C } & 1.208049 & -0.000966 & -0.698808 \\ \text { H } & -0.932697 & 0.000482 & -0.538452 \\ \text { H } & -0.932823 & 0.001279 & 1.933470\end{array}$




$\begin{array}{rrrr}H & 1.207911 & 0.000246 & 3.169563 \\ H & 3.348711 & -0.001477 & 1.933714 \\ H & 3.348840 & -0.002235 & -0.538271 \\ H & 1.208159 & -0.001313 & -1.774332 \\ \text { E= } & -230.7764534197 & \end{array}$

CI $(\pi-\sigma)$ (Dewar Benzene) $1 / 2$

CAS(5,6)/6-31G(d) optimized structure:

$\begin{array}{rrrr}\text { C } & -1.255643 & -0.682965 & -0.308699 \\ \text { C } & -0.000097 & -0.863744 & 0.493873 \\ \text { C } & -1.255539 & 0.683241 & -0.308622 \\ \text { C } & 0.000097 & 0.863743 & 0.493873 \\ \text { C } & 1.255643 & 0.682965 & -0.308699 \\ \text { C } & 1.255539 & -0.683241 & -0.308622 \\ \text { H } & -1.946910 & -1.412192 & -0.686038 \\ \text { H } & -1.946677 & 1.412619 & -0.685905 \\ \text { H } & 0.000186 & 1.487033 & 1.373216 \\ \text { H } & -0.000186 & -1.487033 & 1.373216 \\ \text { H } & 1.946677 & -1.412619 & -0.685906 \\ \text { H } & 1.946910 & 1.412192 & -0.686038 \\ & & \end{array}$

CI (CTS) (Dewar Benzene) 1/2

CAS(5,6)/6-31G(d) optimized structure:

$\begin{array}{rrrr}\text { C } & -1.285226 & -0.673184 & -0.260302 \\ \text { C } & 0.001871 & -1.126693 & 0.322326 \\ \text { C } & -1.286758 & 0.669226 & -0.263540 \\ \text { C } & -0.000733 & 1.128210 & 0.317141 \\ \text { C } & 1.284393 & 0.672185 & -0.267847 \\ \text { C } & 1.285961 & -0.670225 & -0.264609 \\ \text { H } & -2.085840 & -1.342685 & -0.511607 \\ \text { H } & -2.088794 & 1.335773 & -0.518134 \\ \text { H } & -0.000218 & 1.848076 & 1.118963 \\ \text { H } & 0.004043 & -1.843373 & 1.126986 \\ \text { H } & 2.087266 & -1.337883 & -0.518599 \\ \text { H } & 2.084035 & 1.340574 & -0.525125 \\ & & \\ \text { E= } & -230.3366355050 & \end{array}$

1 (Hexamethyl Dewar Benzene) 0/1

RB3LYP/6-311+G(d,p) optimized structure:

$\begin{array}{lrrr}\text { C } & -0.889703 & 2.16646 \odot & 1.723608 \\ \text { C } & -0.282341 & 1.300533 & 0.671460 \\ \text { C } & 0.530979 & -0.000045 & 0.791441 \\ \text { C } & -0.282315 & 1.300545 & -0.671468 \\ \text { C } & 0.530979 & -0.000050 & -0.791441 \\ \text { C } & -0.889638 & 2.166489 & -1.723623 \\ \text { C } & 1.804259 & -0.000168 & -1.617142 \\ \text { C } & -0.890014 & -2.166340 & 1.723621 \\ \text { C } & -0.282561 & -1.300490 & -0.671460 \\ \text { C } & 1.804259 & -0.000135 & 1.617142 \\ \text { C } & -0.282535 & -1.300502 & 0.671468 \\ \text { C } & -0.890079 & -2.166311 & -1.723606 \\ \text { H } & -0.132242 & -2.536764 & -2.423398 \\ \text { H } & -1.624706 & -1.610072 & -2.318620 \\ \text { H } & -1.397405 & -3.032787 & -1.290415 \\ \text { H } & -1.397344 & -3.032816 & 1.290434 \\ \text { H } & -1.624630 & -1.610115 & 2.318662 \\ \text { H } & -0.132154 & -2.536795 & 2.423387 \\ \text { H } & 2.411117 & 0.886877 & 1.411233 \\ \text { H } & 2.410981 & -0.887248 & 1.411258\end{array}$

$\begin{array}{rrrr}H & 1.576568 & -0.000102 & 2.689237 \\ \mathrm{H} & 1.576568 & -0.000137 & -2.689237 \\ \mathrm{H} & 2.410964 & -0.887290 & -1.411252 \\ \mathrm{H} & 2.411134 & 0.886835 & -1.411239 \\ \mathrm{H} & -0.131688 & 2.536934 & -2.423297 \\ \mathrm{H} & -1.396946 & 3.032975 & -1.290431 \\ \mathrm{H} & -1.624236 & 1.610350 & -2.318766 \\ \mathrm{H} & -1.397007 & 3.032946 & 1.290411 \\ \mathrm{H} & -0.131776 & 2.536904 & 2.423308 \\ \mathrm{H} & -1.624312 & 1.610307 & 2.318723\end{array}$

$E(R B+H F-L Y P)=-468.161055019$

$\pi$ RC (Hexamethyl Dewar Benzene) 1/2

UB3LYP/6-311+G(d,p) optimized structure:

$\begin{array}{rrrr}\text { C } & -0.960102 & 2.018527 & 1.727890 \\ \text { C } & -0.291582 & 1.204880 & 0.693344 \\ \text { C } & 0.646690 & -0.000025 & 0.793626 \\ \text { C } & -0.291631 & 1.204825 & -0.693361 \\ \text { C } & 0.646678 & -0.000063 & -0.793616 \\ \text { C } & -0.960191 & 2.018424 & -1.727918 \\ \text { C } & 1.877026 & 0.000331 & -1.669604 \\ \text { C } & -0.959621 & -2.018999 & 1.727871 \\ \text { C } & -0.291191 & -1.205210 & -0.693340 \\ \text { C } & 1.877052 & 0.000209 & 1.669594 \\ \text { C } & -0.291221 & -1.205155 & 0.693407 \\ \text { C } & -0.959538 & -2.019137 & -1.727774 \\ \text { H } & -0.218111 & -2.511164 & -2.367553 \\ \text { H } & -1.555944 & -1.380436 & -2.391747 \\ \text { H } & -1.616318 & -2.777342 & -1.299578 \\ \text { H } & -1.616366 & -2.777247 & 1.299694 \\ \text { H } & -1.556088 & -1.380254 & 2.391748 \\ \text { H } & -0.218233 & -2.510967 & 2.367738 \\ \text { H } & 2.489022 & 0.885444 & 1.481567 \\ \text { H } & 2.489533 & -0.884626 & 1.481343 \\ \text { H } & 1.605124 & 0.000005 & 2.728870 \\ \text { H } & 1.605080 & 0.000941 & -2.728875 \\ \text { H } & 2.489244 & -0.884817 & -1.481980 \\ \text { H } & 2.489265 & 0.885252 & -1.480968 \\ \text { H } & -0.218779 & 2.512565 & -2.366115 \\ \text { H } & -1.619001 & 2.774986 & -1.299931 \\ \text { H } & -1.554294 & 1.379218 & -2.393435 \\ \text { H } & -1.618737 & 2.775233 & 1.299889 \\ \text { H } & -0.218682 & 2.512481 & 2.366219 \\ \text { H } & -1.554401 & 1.379366 & 2.393280 \\ & & & \\ \text { E(UB+HF- LYP })= & -467.895812076 & \\ & & & \end{array}$

$\pi-\sigma$ TS (Hexamethyl Dewar Benzene) $1 / 2$ UB3LYP/6-311+G(d,p) optimized structure:

$\begin{array}{rrrr}\text { C } & 1.348726 & 0.653014 & -0.251637 \\ \text { C } & 0.011912 & 0.861585 & 0.484914 \\ \text { C } & 1.329945 & -0.680273 & -0.277367 \\ \text { C } & -0.012414 & -0.879599 & 0.451560 \\ \text { C } & -1.232906 & -0.672662 & -0.385612 \\ \text { C } & -1.213046 & 0.721540 & -0.359527 \\ \text { C } & -0.071214 & -1.754222 & 1.674602 \\ \text { H } & -1.014238 & -1.655462 & 2.216171 \\ \text { H } & 0.041827 & -2.805624 & 1.384355 \\ \text { H } & 0.754693 & -1.520735 & 2.350313 \\ \text { C } & 2.188298 & -1.752852 & -0.849166 \\ \text { H } & 2.524297 & -2.451133 & -0.076505 \\ \text { H } & 1.647916 & -2.336386 & -1.602739 \\ \text { H } & 3.073065 & -1.325634 & -1.325493 \\ \text { C } & 2.236943 & 1.722403 & -0.782289 \\ \text { H } & 2.587681 & 2.383992 & 0.015679\end{array}$




$\begin{array}{rrrr}\mathrm{H} & 3.112320 & 1.289288 & -1.270499 \\ \mathrm{H} & 1.714878 & 2.345864 & -1.516519 \\ \mathrm{C} & -0.023818 & 1.689476 & 1.740861 \\ \mathrm{H} & -0.966813 & 1.589416 & 2.282283 \\ \mathrm{H} & 0.799537 & 1.413018 & 2.403329 \\ \mathrm{H} & 0.109634 & 2.748726 & 1.490521 \\ \mathrm{C} & -2.211797 & 1.734303 & -0.756077 \\ \mathrm{H} & -2.539774 & 2.320007 & 0.110343 \\ \mathrm{H} & -1.747392 & 2.451978 & -1.445368 \\ \mathrm{H} & -3.084927 & 1.296305 & -1.239380 \\ \mathrm{C} & -2.260332 & -1.641096 & -0.818260 \\ \mathrm{H} & -2.601546 & -2.252044 & 0.025237 \\ \mathrm{H} & -3.122559 & -1.160529 & -1.280167 \\ \mathrm{H} & -1.817528 & -2.342764 & -1.537640\end{array}$

$E(U B+H F-L Y P)=-467.883685301$

$\sigma$ RC (Hexamethyl Dewar Benzene) 1/2

UB3LYP/6-311+G(d,p) optimized structure:

$\begin{array}{rrrr}\text { C } & 1.866688 & 0.00000 \odot & 1.582252 \\ \mathrm{C} & 0.984911 & 0.00000 \odot & 0.376187 \\ \mathrm{C} & -1.866691 & 0.00000 \odot & 1.582253 \\ \mathrm{C} & 0.672502 & 1.299943 & -0.315912 \\ \mathrm{C} & 0.672502 & -1.299943 & -0.315913 \\ \mathrm{C} & -0.672509 & -1.299943 & -0.315913 \\ \mathrm{C} & -0.672509 & 1.299943 & -0.315913 \\ \mathrm{C} & 1.687129 & 2.316661 & -0.714321 \\ \mathrm{C} & 1.687129 & -2.316661 & -0.714322 \\ \mathrm{C} & -1.687136 & -2.316660 & -0.714324 \\ \mathrm{C} & -1.687136 & 2.316660 & -0.714325 \\ \mathrm{C} & -0.984918 & 0.0000 \odot \odot & 0.376186 \\ \mathrm{H} & -2.380232 & 1.900776 & -1.453465 \\ \mathrm{H} & -1.212376 & 3.198653 & -1.145185 \\ \mathrm{H} & -2.287065 & 2.639771 & 0.142581 \\ \mathrm{H} & 2.287057 & 2.639771 & 0.142586 \\ \mathrm{H} & 1.212369 & 3.198655 & -1.145180 \\ \mathrm{H} & 2.380226 & 1.900779 & -1.453461 \\ \mathrm{H} & -2.914516 & 0.00000 \odot & 1.246826 \\ \mathrm{H} & -1.726150 & 0.893844 & 2.192509 \\ \mathrm{H} & -1.726150 & -0.893843 & 2.192510 \\ \mathrm{H} & 1.726149 & 0.893843 & 2.192508 \\ \mathrm{H} & 2.914512 & 0.000000 & 1.246821 \\ \mathrm{H} & 1.726149 & -0.893843 & 2.192508 \\ \mathrm{H} & 1.212369 & -3.198655 & -1.145180 \\ \mathrm{H} & 2.287058 & -2.639770 & 0.142585 \\ \mathrm{H} & 2.380226 & -1.900778 & -1.453462 \\ \mathrm{H} & -2.380232 & -1.900777 & -1.453464 \\ \mathrm{H} & -2.287064 & -2.639772 & 0.142581 \\ \mathrm{H} & -1.212375 & -3.198653 & -1.145184 \\ & & & \\ \mathrm{E}(\mathrm{UB}+\mathrm{HF}-\mathrm{LYP})= & -467.888325781 & \\ \mathrm{H} & & & \end{array}$

CTS (Hexamethyl Dewar Benzene) 1/2

UB3LYP/6-311+G(d,p) optimized structure:

$\begin{array}{rr}\text { C } & -1.256897 \\ \text { C } & 0.031793 \\ \text { C } & -1.309613 \\ \text { C } & -0.037228 \\ \text { C } & 1.273226 \\ \text { C } & 1.305523 \\ \text { C } & 0.049215 \\ \text { H } & 0.945958 \\ H & -0.857200 \\ \text { H } & 0.073401 \\ \text { C } & -2.234456 \\ \text { H } & -3.120986\end{array}$

-0.770847
-1.041324
0.571756
1.160305
0.653258
-0.689901
-1.773657
-1.573509
-1.615719
-2.847129
-1.844529
-1.421539

-0.219848
0.478779
-0.316202
0.209162
-0.308192
-0.210837
1.760855
2.348654
2.346903
1.498591
-0.575409
-1.047570

$\begin{array}{lrrr}\text { H } & -1.791198 & -2.562944 & -1.273345 \\ \text { H } & -2.557457 & -2.406242 & 0.307151 \\ \text { C } & -2.449200 & 1.437189 & -0.751449 \\ \text { H } & -2.778446 & 2.097718 & 0.056199 \\ \text { H } & -2.141270 & 2.079131 & -1.584041 \\ \text { H } & -3.305682 & 0.844740 & -1.072036 \\ \text { C } & -0.077549 & 2.365782 & 1.090034 \\ \text { H } & 0.805864 & 2.436450 & 1.727176 \\ \text { H } & -0.100252 & 3.278371 & 0.474048 \\ \text { H } & -0.971209 & 2.384795 & 1.716339 \\ \text { C } & 2.357558 & 1.589195 & -0.738241 \\ \text { H } & 2.629089 & 2.279952 & 0.065321 \\ \text { H } & 3.256783 & 1.053083 & -1.040266 \\ \text { H } & 2.018200 & 2.199033 & -1.582796 \\ \text { C } & 2.352170 & -1.699300 & -0.558011 \\ \text { H } & 2.696134 & -2.245748 & 0.326141 \\ \text { H } & 1.963607 & -2.439080 & -1.266166 \\ \text { H } & 3.217750 & -1.219872 & -1.014994\end{array}$

$E(U B+H F-L Y P)=-467.884839765$

BRC (Hexamethylbenzene) $1 / 2$ UB3LYP/6-311+G(d,p) optimized structure:

$\begin{array}{rrrr}\text { C } & -1.205320 & -0.017931 & -0.698131 \\ \text { C } & -1.228537 & 0.032044 & 0.742035 \\ \text { C } & -0.033019 & -0.029353 & 1.434664 \\ \text { C } & 1.205319 & 0.017931 & 0.698132 \\ \text { C } & 1.228537 & -0.032044 & -0.742034 \\ \text { C } & 0.033019 & 0.029354 & -1.434664 \\ \text { C } & -2.552270 & 0.124877 & 1.457200 \\ \text { H } & -3.269327 & 0.724288 & 0.896191 \\ \text { H } & -2.998685 & -0.866119 & 1.603296 \\ \text { H } & -2.444344 & 0.582957 & 2.438197 \\ \text { C } & -2.490741 & -0.160605 & -1.443341 \\ \text { H } & -2.894006 & 0.836757 & -1.679129 \\ \text { H } & -2.361757 & -0.678935 & -2.392028 \\ \text { H } & -3.250885 & -0.676125 & -0.858784 \\ \text { C } & -0.005024 & 0.121619 & -2.938476 \\ \text { H } & 0.899884 & 0.579462 & -3.332430 \\ \text { H } & -0.100677 & -0.869263 & -3.398607 \\ \text { H } & -0.848177 & 0.721427 & -3.281099 \\ \text { C } & 2.552269 & -0.124874 & -1.457200 \\ \text { H } & 2.998678 & 0.866124 & -1.603305 \\ \text { H } & 3.269331 & -0.724277 & -0.896189 \\ \text { H } & 2.444344 & -0.582962 & -2.438194 \\ \text { C } & 2.490742 & 0.160602 & 1.443339 \\ \text { H } & 2.361759 & 0.678919 & 2.392034 \\ \text { H } & 2.894015 & -0.836760 & 1.679112 \\ \text { H } & 3.250880 & 0.676135 & 0.858786 \\ \text { C } & 0.005024 & -0.121619 & 2.938476 \\ \text { H } & 0.100698 & 0.869261 & 3.398607 \\ \text { H } & -0.899889 & -0.579446 & 3.332433 \\ \text { H } & 0.848168 & -0.721443 & 3.281097\end{array}$

$E(U B+H F-L Y P)=-467.978753560$

2 (Hexamethylbenzene) 0/1 RB3LYP/6-311+G(d,p) optimized structure:

$\begin{array}{rrrr}C & -1.217091 & -0.010927 & -0.705098 \\ C & -1.219347 & 0.011706 & 0.701337 \\ C & -0.002221 & -0.010927 & 1.406342 \\ C & 1.217075 & 0.010964 & 0.705121 \\ C & 1.219332 & -0.011670 & -0.701321 \\ C & 0.002209 & 0.010961 & -1.406324 \\ C & -2.532722 & 0.083670 & 1.458332\end{array}$




$\begin{array}{rrrr}\mathrm{H} & -3.296739 & 0.610086 & 0.886024 \\ \mathrm{H} & -2.931190 & -0.911545 & 1.693732 \\ \mathrm{H} & -2.421987 & 0.618056 & 2.402127 \\ \mathrm{C} & -2.527895 & -0.081874 & -1.466618 \\ \mathrm{H} & -2.921742 & 0.913602 & -1.708596 \\ \mathrm{H} & -2.415272 & -0.621321 & -2.407367 \\ \mathrm{H} & -3.296063 & -0.602650 & -0.894810 \\ \mathrm{C} & 0.004431 & 0.082475 & -2.921894 \\ \mathrm{H} & 0.879980 & 0.613288 & -3.296198 \\ \mathrm{H} & 0.005826 & -0.912861 & -3.384479 \\ \mathrm{H} & -0.870762 & 0.611998 & -3.298836 \\ \mathrm{C} & 2.532660 & -0.083647 & -1.458399 \\ \mathrm{H} & 2.931129 & 0.911565 & -1.693811 \\ \mathrm{H} & 3.296711 & -0.610105 & -0.886184 \\ \mathrm{H} & 2.421824 & -0.617996 & -2.402207 \\ \mathrm{C} & 2.527838 & 0.081911 & 1.466714 \\ \mathrm{H} & 2.415084 & 0.621108 & 2.407593 \\ \mathrm{H} & 2.921811 & -0.913568 & 1.708475 \\ \mathrm{H} & 3.295962 & 0.602942 & 0.895083 \\ \mathrm{C} & -0.004359 & -0.082455 & 2.921912 \\ \mathrm{H} & -0.005777 & 0.912876 & 3.384507 \\ \mathrm{H} & -0.879836 & -0.613335 & 3.296277 \\ \mathrm{H} & 0.870904 & -0.611921 & 3.298780\end{array}$

$E(R B+H F-L Y P)=-468.255505703$

1 (Hexamethyl Dewar Benzene) 0/1 CAS(6,6)/6-31G(d) optimized structure:

$\begin{array}{rrrr}\text { C } & -0.910990 & 2.143736 & 1.723887 \\ \mathrm{C} & -0.272926 & 1.289903 & 0.672976 \\ \mathrm{C} & 0.538033 & -0.000077 & 0.801938 \\ \mathrm{C} & -0.272997 & 1.289870 & -0.672955 \\ \mathrm{C} & 0.538033 & -0.000065 & -0.801940 \\ \mathrm{C} & -0.911178 & 2.143647 & -1.723840 \\ \mathrm{C} & 1.800466 & -0.000339 & -1.641606 \\ \mathrm{C} & -0.911800 & -2.143672 & 1.723745 \\ \mathrm{C} & -0.273493 & -1.289789 & -0.673003 \\ \mathrm{C} & 1.800468 & -0.000440 & 1.641601 \\ \mathrm{C} & -0.273571 & -1.289749 & 0.672984 \\ \mathrm{C} & -0.911603 & -2.143773 & -1.723787 \\ \mathrm{H} & -0.173352 & -2.519775 & -2.427446 \\ \mathrm{H} & -1.641613 & -1.576948 & -2.296974 \\ \mathrm{H} & -1.420423 & -2.997595 & -1.288609 \\ \mathrm{H} & -1.420608 & -2.997496 & 1.288556 \\ \mathrm{H} & -1.641844 & -1.576804 & 2.296846 \\ \mathrm{H} & -0.173623 & -2.519669 & 2.427483 \\ \mathrm{H} & 2.405843 & 0.878895 & 1.442007 \\ \mathrm{H} & 2.404597 & -0.880875 & 1.443108 \\ \mathrm{H} & 1.561698 & 0.000401 & 2.702731 \\ \mathrm{H} & 1.561694 & 0.000511 & -2.702735 \\ \mathrm{H} & 2.404648 & -0.880741 & -1.443132 \\ \mathrm{H} & 2.405789 & 0.879028 & -1.441994 \\ \mathrm{H} & -0.172689 & 2.521404 & -2.426318 \\ \mathrm{H} & -1.422051 & 2.996274 & -1.288741 \\ \mathrm{H} & -1.639412 & 1.575875 & -2.298357 \\ \mathrm{H} & -1.421873 & 2.996364 & 1.288802 \\ \mathrm{H} & -0.172429 & 2.521493 & 2.426289 \\ \mathrm{H} & -1.639193 & 1.576006 & 2.298484 \\ & & & \\ \mathrm{E}= & -464.8843705277 & & \end{array}$

$\pi$ RC (Hexamethyl Dewar Benzene) 1/2 CAS(5,6)/6-31G(d) optimized structure:

$\begin{array}{rrrr}\text { C } & -0.921050 & 2.082218 & 1.724015 \\ \text { C } & -0.292693 & 1.211146 & 0.705440 \\ \text { C } & 0.600449 & -0.002510 & 0.809613\end{array}$

$\begin{array}{rrrr}\text { C } & -0.292673 & 1.211161 & -0.705446 \\ \text { C } & 0.600445 & -0.002513 & -0.809623 \\ \text { C } & -0.920992 & 2.082261 & -1.724020 \\ \text { C } & 1.822958 & 0.011316 & -1.701309 \\ \text { C } & -0.933073 & -2.075942 & 1.737068 \\ \text { C } & -0.280840 & -1.250598 & -0.675918 \\ \text { C } & 1.822966 & 0.011333 & 1.701295 \\ \text { C } & -0.280830 & -1.250600 & 0.675924 \\ \text { C } & -0.933100 & -2.075944 & -1.737049 \\ \text { H } & -0.192446 & -2.495862 & -2.409473 \\ \text { H } & -1.616713 & -1.480033 & -2.335476 \\ \text { H } & -1.495863 & -2.892587 & -1.302493 \\ \text { H } & -1.495841 & -2.892588 & 1.302527 \\ \text { H } & -1.616678 & -1.480028 & 2.335503 \\ \text { H } & -0.192408 & -2.495854 & 2.409484 \\ \text { H } & 2.426679 & 0.897738 & 1.540203 \\ \text { H } & 2.437002 & -0.858513 & 1.501463 \\ \text { H } & 1.536191 & -0.017158 & 2.747345 \\ \text { H } & 1.536179 & -0.017172 & -2.747358 \\ \text { H } & 2.436985 & -0.858537 & -1.501479 \\ \text { H } & 2.426682 & 0.897714 & -1.540220 \\ \text { H } & -0.150984 & 2.525738 & -2.349005 \\ \text { H } & -1.523316 & 2.867143 & -1.287833 \\ \text { H } & -1.544466 & 1.475770 & -2.376116 \\ \text { H } & -1.523379 & 2.867096 & 1.287826 \\ \text { H } & -0.151063 & 2.525700 & 2.349021 \\ \text { H } & -1.544526 & 1.475707 & 2.376089 \\ & & & \\ \text { E= } & -464.6180128040 & & \\ & & & \end{array}$

$\pi-\sigma$ TS (Hexamethyl Dewar Benzene) 1/2 CAS(5,6)/6-31G(d) optimized structure:

$\begin{array}{lrrr}\text { C } & -1.343240 & -0.667905 & -0.233376 \\ \text { C } & -0.004591 & -0.907908 & 0.455602 \\ \text { C } & -1.343119 & 0.668119 & -0.233445 \\ \text { C } & -0.004424 & 0.907946 & 0.455506 \\ \text { C } & 1.200771 & 0.694484 & -0.379671 \\ \text { C } & 1.200649 & -0.694749 & -0.379589 \\ \text { C } & 0.069293 & 1.801953 & 1.668531 \\ \text { H } & 1.002882 & 1.688506 & 2.206280 \\ \text { H } & -\Theta .021626 & 2.841193 & 1.363683 \\ \text { H } & -0.750075 & 1.585562 & 2.342868 \\ \text { C } & -2.242824 & 1.728238 & -0.787083 \\ \text { H } & -2.572776 & 2.407968 & -0.008561 \\ \text { H } & -1.732390 & 2.315435 & -1.544918 \\ \text { H } & -3.121430 & 1.286151 & -1.239477 \\ \text { C } & -2.243141 & -1.727915 & -0.786904 \\ \text { H } & -2.573219 & -2.407503 & -0.008312 \\ \text { H } & -3.121665 & -1.285714 & -1.239345 \\ \text { H } & -1.732815 & -2.315286 & -1.544677 \\ \text { C } & 0.068956 & -1.801808 & 1.668716 \\ \text { H } & 1.002560 & -1.688476 & 2.206462 \\ \text { H } & -0.750379 & -1.585203 & 2.343025 \\ \text { H } & -0.022148 & -2.841062 & 1.363971 \\ \text { C } & 2.242675 & -1.680196 & -0.782105 \\ \text { H } & 2.661989 & -2.162975 & 0.095481 \\ \text { H } & 1.790871 & -2.456805 & -1.391624 \\ \text { H } & 3.044749 & -1.217706 & -1.340149 \\ \text { C } & 2.242965 & 1.679702 & -0.782312 \\ \text { H } & 2.662433 & 2.162440 & 0.095223 \\ \text { H } & 3.044912 & 1.217021 & -1.340382 \\ \text { H } & 1.791279 & 2.456371 & -1.391841 \\ & & & \\ \text { E= } & -464.6099522723 & & \\ & & & \end{array}$

$\sigma$ RC (Hexamethyl Dewar Benzene) 1/2 CAS(5,6)/6-31G(d) optimized structure: 


$\begin{array}{rrrr}\text { C } & 1.857114 & 0.000039 & 1.630484 \\ \text { C } & 0.986169 & 0.000010 & 0.414109 \\ \text { C } & -1.857306 & -0.000028 & 1.630362 \\ \text { C } & 0.661528 & 1.291940 & -0.283273 \\ \text { C } & 0.661542 & -1.291965 & -0.283165 \\ \text { C } & -0.661419 & -1.291958 & -0.283130 \\ \text { C } & -0.661430 & 1.291906 & -0.283169 \\ \text { C } & 1.687401 & 2.287571 & -0.728189 \\ \text { C } & 1.687459 & -2.287656 & -0.727877 \\ \text { C } & -1.687332 & -2.287574 & -0.728013 \\ \text { C } & -1.687364 & 2.287659 & -0.727742 \\ \text { C } & -0.986065 & -0.000006 & 0.414167 \\ \text { H } & -2.354023 & 1.843753 & -1.461511 \\ \text { H } & -1.215629 & 3.152399 & -1.174667 \\ \text { H } & -2.290812 & 2.623489 & 0.109795 \\ \text { H } & 2.291367 & 2.623168 & 0.109072 \\ \text { H } & 1.215623 & 3.152462 & -1.174784 \\ \text { H } & 2.353581 & 1.843582 & -1.462332 \\ \text { H } & -2.894963 & -0.000162 & 1.294171 \\ \text { H } & -1.705898 & 0.887563 & 2.231659 \\ \text { H } & -1.705706 & -0.887530 & 2.231733 \\ \text { H } & 1.705464 & 0.887592 & 2.231780 \\ \text { H } & 2.894871 & 0.000025 & 1.294613 \\ \text { H } & 1.705442 & -0.887496 & 2.231797 \\ \text { H } & 1.215720 & -3.152515 & -1.174580 \\ \text { H } & 2.291207 & -2.623304 & 0.109517 \\ \text { H } & 2.353833 & -1.843724 & -1.461881 \\ \text { H } & -2.353775 & -1.843478 & -1.461870 \\ \text { H } & -2.291025 & -2.623484 & 0.109314 \\ \text { H } & -1.215575 & -3.152278 & -1.174988 \\ & & & \\ \text { E= } & -464.6694092808 & & \\ & & & \end{array}$

CTS (Hexamethyl Dewar Benzene) 1/2

CAS(5,6)/6-31G(d) optimized structure:

$\begin{array}{rrrr}\text { C } & -1.258104 & -0.745807 & -0.211701 \\ \text { C } & 0.029775 & -1.000666 & 0.501094 \\ \text { C } & -1.303306 & 0.593451 & -0.333141 \\ \text { C } & -0.036508 & 1.129618 & 0.261868 \\ \text { C } & 1.265819 & 0.674434 & -0.323827 \\ \text { C } & 1.304171 & -0.665040 & -0.202417 \\ \text { C } & 0.047835 & -1.723040 & 1.802294 \\ \text { H } & 0.928496 & -1.487525 & 2.384548 \\ \text { H } & -0.850153 & -1.543699 & 2.378058 \\ \text { H } & 0.082347 & -2.788244 & 1.565472 \\ \text { C } & -2.228994 & -1.836633 & -0.561814 \\ \text { H } & -3.088405 & -1.431015 & -1.077188 \\ \text { H } & -1.765651 & -2.572925 & -1.210742 \\ \text { H } & -2.581591 & -2.349901 & 0.326768 \\ \text { C } & -2.402571 & 1.485957 & -0.832848 \\ \text { H } & -2.783711 & 2.117430 & -0.037603 \\ \text { H } & -2.033562 & 2.134183 & -1.621077 \\ \text { H } & -3.228001 & 0.909219 & -1.226840 \\ \text { C } & -0.076470 & 2.287600 & 1.215940 \\ \text { H } & 0.802270 & 2.311762 & 1.848083 \\ \text { H } & -0.103855 & 3.220052 & 0.654308 \\ \text { H } & -0.959519 & 2.256194 & 1.841728 \\ \text { C } & 2.310290 & 1.634511 & -0.815753 \\ \text { H } & 2.645131 & 2.288549 & -0.017908 \\ \text { H } & 3.173238 & 1.110990 & -1.203617 \\ \text { H } & 1.906916 & 2.258395 & -1.606783 \\ \text { C } & 2.344363 & -1.692469 & -0.545202 \\ \text { H } & 2.722422 & -2.182408 & 0.346078 \\ \text { H } & 1.932941 & -2.456571 & -1.196968 \\ \text { H } & 3.180050 & -1.233475 & -1.054784 \\ & & & \\ \text { E } & -464.6198182189 & & \\ & & & \end{array}$

BRC (Hexamethylbenzene) 1/2

CAS(5,6)/6-31G(d) optimized structure:

\begin{tabular}{|c|c|c|c|}
\hline C & $\odot .0 \odot 4035$ & -0.018294 & ๑.0๑९185 \\
\hline C & -0.017656 & 0.028164 & 1.437684 \\
\hline C & 1.175532 & -0.027417 & 2.128946 \\
\hline C & 2.411881 & 0.017645 & 1.395002 \\
\hline C & 2.433572 & -0.028806 & $-0.04250 \odot$ \\
\hline C & 1.240389 & $\odot .026770$ & -0.733761 \\
\hline C & -1.346781 & 0.125572 & 2.157186 \\
\hline $\mathrm{H}$ & -2.054145 & 0.716435 & 1.592797 \\
\hline $\mathrm{H}$ & -1.782396 & -0.856420 & 2.316376 \\
\hline $\mathrm{H}$ & -1.233681 & $\odot .601209$ & 3.119165 \\
\hline C & -1.292987 & -0.151769 & $-\odot .751407$ \\
\hline $\mathrm{H}$ & -1.688959 & $\odot .834432$ & -0.984953 \\
\hline $\mathrm{H}$ & -1.158864 & -0.682171 & -1.681842 \\
\hline $\mathrm{H}$ & -2.035832 & -0.676395 & -0.170179 \\
\hline C & 1.203759 & $\odot .122603$ & -2.244813 \\
\hline $\mathrm{H}$ & 2.093003 & $\odot .601388$ & -2.624980 \\
\hline $\mathrm{H}$ & 1.129422 & -0.860189 & -2.700907 \\
\hline $\mathrm{H}$ & 0.360303 & $\odot .709752$ & -2.579074 \\
\hline C & 3.762667 & -0.126191 & -0.762057 \\
\hline $\mathrm{H}$ & 4.198290 & $\odot .855806$ & -0.921197 \\
\hline $\mathrm{H}$ & 4.470050 & -0.717114 & $-\odot .197757$ \\
\hline $\mathrm{H}$ & 3.649501 & -0.601746 & -1.724072 \\
\hline C & 3.708883 & $\odot .151077$ & 2.146635 \\
\hline $\mathrm{H}$ & 3.574691 & $\odot .681244$ & 3.077194 \\
\hline $\mathrm{H}$ & 4.104950 & -0.835139 & 2.379959 \\
\hline $\mathrm{H}$ & 4.451682 & 0.675915 & 1.565541 \\
\hline C & 1.212236 & -0.123265 & 3.639996 \\
\hline $\mathrm{H}$ & 1.286587 & 0.859521 & 4.096099 \\
\hline $\mathrm{H}$ & 0.323038 & -0.602082 & 4.020221 \\
\hline $\mathrm{H}$ & 2.055738 & -0.710396 & 3.97418 \\
\hline & & & \\
\hline
\end{tabular}

2 (Hexamethylbenzene) 0/1

CAS(6,6)/6-31G(d) optimized structure:

$\begin{array}{rrrr}\text { C } & -0.009176 & -0.012473 & -0.007786 \\ \text { C } & -0.011454 & 0.012845 & 1.398962 \\ \text { C } & 1.205684 & -0.011670 & 2.104349 \\ \text { C } & 2.425133 & 0.011876 & 1.402937 \\ \text { C } & 2.427420 & -0.013500 & -0.003820 \\ \text { C } & 1.210272 & 0.011102 & -0.709211 \\ \text { C } & -1.327047 & 0.095365 & 2.155678 \\ \text { H } & -2.077793 & 0.626677 & 1.586559 \\ \text { H } & -1.728604 & -0.888917 & 2.387781 \\ \text { H } & -1.212931 & 0.628342 & 3.089888 \\ \text { C } & -1.322407 & -0.094043 & -0.768704 \\ \text { H } & -1.723339 & 0.890525 & -1.000662 \\ \text { H } & -1.205413 & -0.625712 & -1.703288 \\ \text { H } & -2.074842 & -0.626212 & -0.202596 \\ \text { C } & 1.212710 & 0.091863 & -2.227005 \\ \text { H } & 2.081258 & 0.622211 & -2.593276 \\ \text { H } & 1.211814 & -0.892943 & -2.689698 \\ \text { H } & 0.346921 & 0.624977 & -2.595837 \\ \text { C } & 3.743036 & -0.096248 & -0.760485 \\ \text { H } & 4.144975 & 0.887950 & -0.992270 \\ \text { H } & 4.493541 & -0.627989 & -0.191439 \\ \text { H } & 3.628824 & -0.628937 & -1.694841 \\ \text { C } & 3.738348 & 0.093409 & 2.163892 \\ \text { H } & 3.621459 & 0.625662 & 3.098161 \\ \text { H } & 4.138910 & -0.891166 & 2.396457 \\ \text { H } & 4.491025 & 0.624976 & 1.597554 \\ \text { C } & 1.203151 & -0.092321 & 3.622147 \\ \text { H } & 1.203034 & 0.892524 & 4.084755 \\ & & & \end{array}$




$\begin{array}{rrrr}H & 0.335021 & -0.623443 & 3.988316 \\ H & 2.069376 & -0.624590 & 3.991157 \\ \text { E }= & -464.9736569625 & \end{array}$

CI $(\pi-\sigma)$ (Hexamethyl Dewar Benzene) 1/2 CAS(5,6)/6-31G(d) optimized structure:

\begin{tabular}{|c|c|c|c|}
\hline C & -0.857718 & 2.190439 & 1.720783 \\
\hline C & -0.334233 & 1.255208 & 0.684276 \\
\hline C & 0.478929 & -0.000101 & 0.872043 \\
\hline C & -0.334347 & 1.255154 & -0.684243 \\
\hline C & 0.478929 & $-\odot . \odot \odot \odot \odot 74$ & -0.872043 \\
\hline C & -0.857986 & 2.190320 & -1.720733 \\
\hline C & 1.698644 & $\odot .000126$ & -1.761064 \\
\hline C & -0.857457 & -2.190818 & 1.720733 \\
\hline C & -0.333930 & -1.255579 & -0.684276 \\
\hline C & 1.698644 & -๑.๑๑९९९७ & 1.761064 \\
\hline C & $-\odot .334044$ & -1.255525 & 0.684243 \\
\hline C & -0.857189 & -2.190937 & -1.720783 \\
\hline $\mathrm{H}$ & -0.043390 & -2.593987 & -2.315378 \\
\hline $\mathrm{H}$ & -1.526090 & -1.667454 & -2.397651 \\
\hline $\mathrm{H}$ & -1.396193 & -3.015738 & -1.273892 \\
\hline $\mathrm{H}$ & -1.396438 & -3.015620 & 1.273819 \\
\hline $\mathrm{H}$ & -1.526414 & -1.667279 & 2.397502 \\
\hline $\mathrm{H}$ & -0.043740 & -2.593869 & 2.315438 \\
\hline $\mathrm{H}$ & 2.306594 & ๑.880131 & 1.589357 \\
\hline $\mathrm{H}$ & 2.306735 & $-\odot .880042$ & 1.589332 \\
\hline $\mathrm{H}$ & 1.398373 & -0.000045 & 2.805161 \\
\hline $\mathrm{H}$ & 1.398373 & 0.000091 & -2.805161 \\
\hline $\mathrm{H}$ & 2.306807 & -0.879865 & -1.589357 \\
\hline $\mathrm{H}$ & 2.306523 & $\odot .880308$ & -1.589333 \\
\hline $\mathrm{H}$ & $-\odot .044367$ & 2.593563 & -2.315442 \\
\hline $\mathrm{H}$ & -1.397162 & 3.014995 & -1.273819 \\
\hline $\mathrm{H}$ & -1.526821 & 1.666620 & -2.397499 \\
\hline $\mathrm{H}$ & -1.396917 & 3. 015112 & 1.273892 \\
\hline $\mathrm{H}$ & -0.044018 & 2.593681 & 2.315382 \\
\hline $\mathrm{H}$ & -1.526497 & 1.666795 & 2.397648 \\
\hline
\end{tabular}

CI (CTS) (Hexamethyl Dewar Benzene) 1/2 CAS(5,6)/6-31G(d) optimized structure:

$\begin{array}{rr}\mathrm{C} & -1.258180 \\ \mathrm{C} & 0.032182 \\ \mathrm{C} & -1.299782 \\ \mathrm{C} & -0.038655 \\ \mathrm{C} & 1.260564 \\ \mathrm{C} & 1.302173 \\ \mathrm{C} & 0.057341 \\ \mathrm{H} & 0.934756 \\ \mathrm{H} & -0.832417 \\ \mathrm{H} & 0.090982 \\ \mathrm{C} & -2.302416 \\ \mathrm{H} & -3.157291 \\ \mathrm{H} & -1.895956 \\ \mathrm{H} & -2.651755 \\ \mathrm{C} & -2.403736 \\ \mathrm{H} & -2.787864 \\ \mathrm{H} & -2.041133 \\ \mathrm{H} & -3.226894 \\ \mathrm{C} & -0.079369 \\ \mathrm{H} & 0.801126 \\ \mathrm{H} & -0.105642 \\ \mathrm{H} & -0.966046 \\ \mathrm{C} & 2.310905 \\ \mathrm{H} & 2.647569\end{array}$

$\begin{array}{rrrr}\text { H } & 3.172289 & 1.107516 & -1.196717 \\ \text { H } & 1.915378 & 2.247583 & -1.636707 \\ \mathrm{C} & 2.412063 & -1.613237 & -0.424610 \\ \mathrm{H} & 2.783456 & -2.056910 & 0.492946 \\ \mathrm{H} & 2.060205 & -2.418093 & -1.062079 \\ \mathrm{H} & 3.241812 & -1.130819 & -0.921076 \\ \mathrm{H} & & \\ \mathrm{E}= & -464.6122768545 & \end{array}$

$30 / 1$

RB3LYP/6-311+G(d,p) optimized structure:

\begin{tabular}{|c|c|c|c|}
\hline $\mathrm{C}$ & 2.508072 & -1.757565 & $\odot .183544$ \\
\hline $\mathrm{C}$ & 3.451877 & -1.233824 & -0.897989 \\
\hline $\mathrm{H}$ & 4.174487 & -0.548608 & -0.435555 \\
\hline $\mathrm{C}$ & 2.652117 & -0.511300 & -2.012726 \\
\hline $\mathrm{H}$ & 4.039626 & -2.061271 & -1.314668 \\
\hline $\mathrm{H}$ & 3.087036 & 0.472034 & -2.227486 \\
\hline C & 1.174904 & -0.381034 & -1.627636 \\
\hline $\mathrm{H}$ & 2.707091 & -1.076470 & -2.952688 \\
\hline $\mathrm{C}$ & 0.770450 & 0.116899 & -0.201673 \\
\hline C & $\odot .435762$ & -1.697369 & -1.304228 \\
\hline $\mathrm{H}$ & $\odot .621921$ & $\odot .188506$ & -2.384259 \\
\hline C & 1.575180 & -0.736179 & ๑.897505 \\
\hline $\mathrm{H}$ & 3.058654 & -2.328578 & $\odot .942541$ \\
\hline $\mathrm{C}$ & 1.231244 & -2.524710 & -0.237955 \\
\hline $\mathrm{C}$ & $\odot .560594$ & 1.579785 & $\odot .063524$ \\
\hline C & 2.090344 & -0.024671 & 2.131082 \\
\hline C & -1.850281 & -0.731198 & -0.257095 \\
\hline $\mathrm{C}$ & $-\odot .046728$ & -2.504167 & 2.166187 \\
\hline $\mathrm{H}$ & $-\odot .053986$ & -2.254510 & -2.107148 \\
\hline $\mathrm{C}$ & $-\odot .383743$ & -0.988156 & $-0.15460 \odot$ \\
\hline C & 0.425229 & -1.816352 & 0.908620 \\
\hline $\mathrm{H}$ & 1.262934 & -3.610009 & -0.374015 \\
\hline $\mathrm{C}$ & 0.203116 & 4.331852 & $\odot .596764$ \\
\hline $\mathrm{C}$ & -0.341149 & 2.017488 & 1.049075 \\
\hline C & 1.269160 & 2.555777 & -0.654662 \\
\hline C & 1.096133 & 3.915725 & -0.390971 \\
\hline $\mathrm{C}$ & $-\odot .517203$ & 3.375043 & 1.314477 \\
\hline $\mathrm{H}$ & $-\odot .924673$ & 1.286130 & 1.600986 \\
\hline $\mathrm{H}$ & 1.964975 & 2.251021 & -1.430910 \\
\hline $\mathrm{H}$ & 1.659749 & 4.649860 & -0.961443 \\
\hline $\mathrm{H}$ & -1.224815 & 3.685459 & 2.079080 \\
\hline $\mathrm{H}$ & 0.064928 & 5.390228 & 0.801340 \\
\hline $\mathrm{C}$ & -4.629314 & -0.302055 & $-\odot .559702$ \\
\hline $\mathrm{C}$ & -2.786978 & -1.535633 & $\odot .412458$ \\
\hline C & -2.342861 & $\odot .295068$ & -1.084766 \\
\hline $\mathrm{C}$ & -3.712334 & $\odot .505503$ & -1.235773 \\
\hline $\mathrm{C}$ & -4.158839 & -1.323629 & $\odot .264897$ \\
\hline $\mathrm{H}$ & -2.443520 & -2.340856 & 1.052470 \\
\hline $\mathrm{H}$ & -1.646044 & 0.941899 & -1.609086 \\
\hline $\mathrm{H}$ & -4.063446 & 1.307643 & -1.880108 \\
\hline $\mathrm{H}$ & -4.860042 & -1.961759 & $\odot .797143$ \\
\hline $\mathrm{H}$ & -5.697156 & -0.135497 & -0.674110 \\
\hline $\mathrm{H}$ & 1.311450 & 0.552326 & 2.640286 \\
\hline $\mathrm{H}$ & 2.893233 & $\odot .677600$ & 1.872089 \\
\hline $\mathrm{H}$ & 2.497985 & -0.748260 & 2.847829 \\
\hline $\mathrm{H}$ & $-\odot .590950$ & -1.820455 & 2.829421 \\
\hline $\mathrm{H}$ & 0.809209 & -2.897783 & 2.730158 \\
\hline $\mathrm{H}$ & -0.704619 & -3.354368 & 1.94845 \\
\hline
\end{tabular}

$31 / 2$

UB3LYP/6-311+G(d,p) optimized structure:

$\begin{array}{lrrr}\text { C } & 1.610940 & -2.526626 & 0.145266 \\ \text { C } & 2.656725 & -2.389502 & -0.958726\end{array}$




\begin{tabular}{|c|c|c|c|}
\hline $\mathrm{H}$ & 3.591179 & -2.016135 & -0.522293 \\
\hline $\mathrm{C}$ & 2.144176 & -1.427439 & -2.058949 \\
\hline $\mathrm{H}$ & 2.884523 & -3.375527 & -1.378004 \\
\hline $\mathrm{H}$ & 2.923572 & -0.719070 & -2.356941 \\
\hline C & $\odot .900388$ & -0.665598 & -1.539789 \\
\hline $\mathrm{H}$ & 1.852289 & -1.973624 & -2.963722 \\
\hline C & 1.132282 & -0.076955 & -0.150910 \\
\hline $\mathrm{C}$ & $-\odot .286964$ & -1.616653 & -1.286170 \\
\hline $\mathrm{H}$ & 0.587609 & 0.107321 & -2.244419 \\
\hline $\mathrm{C}$ & 1.154900 & -1.203475 & ๑.899233 \\
\hline $\mathrm{H}$ & 1.909377 & -3.265721 & ๑. 894824 \\
\hline $\mathrm{C}$ & $\odot .147609$ & -2.729187 & -0.265416 \\
\hline $\mathrm{C}$ & 1.475394 & 1.301694 & ๑. .027971 \\
\hline $\mathrm{C}$ & 1.827967 & -0.963837 & 2.245081 \\
\hline $\mathrm{C}$ & -2.272695 & -0.201260 & -0.198172 \\
\hline $\mathrm{C}$ & -0.963520 & -2.260538 & 2.181969 \\
\hline $\mathrm{H}$ & -0.802848 & -1.934329 & -2.196097 \\
\hline C & -1.087918 & -0.965257 & -0.166381 \\
\hline C & -0.350020 & -1.774382 & ๑. 886520 \\
\hline $\mathrm{H}$ & -0.278772 & -3.722170 & -0.420858 \\
\hline $\mathrm{C}$ & 2.141717 & 4.042962 & ๑. 297718 \\
\hline $\mathrm{C}$ & 1.386045 & 1.969089 & 1. 283878 \\
\hline C & 1.893592 & 2.082969 & -1.091075 \\
\hline $\mathrm{C}$ & 2.237645 & 3.417937 & -0.951251 \\
\hline C & 1.703736 & 3.312623 & 1.408601 \\
\hline $\mathrm{H}$ & 1.024158 & 1.438228 & 2.152655 \\
\hline $\mathrm{H}$ & 1.988565 & 1.621940 & -2.067217 \\
\hline $\mathrm{H}$ & 2.579291 & 3.978889 & -1.815694 \\
\hline $\mathrm{H}$ & 1.609972 & 3.798365 & 2.375068 \\
\hline $\mathrm{H}$ & 2.400657 & 5.092182 & 0.403292 \\
\hline C & -4.596361 & 1.375357 & -0.315647 \\
\hline C & -2.919493 & ๑. 233965 & ๑. 995151 \\
\hline C & -2.832892 & ๑. 183530 & -1.451393 \\
\hline $\mathrm{C}$ & -3.977985 & $\odot .960062$ & -1.503652 \\
\hline C & -4.063451 & 1.011195 & $\odot .929742$ \\
\hline $\mathrm{H}$ & -2.508412 & $-\odot .043720$ & 1.958747 \\
\hline $\mathrm{H}$ & -2.358352 & -0.144984 & -2.370689 \\
\hline $\mathrm{H}$ & -4.399718 & 1.243784 & -2.462795 \\
\hline $\mathrm{H}$ & -4.550413 & 1.338042 & 1.843210 \\
\hline $\mathrm{H}$ & -5.496316 & 1.981561 & -0.359994 \\
\hline $\mathrm{H}$ & 1.230461 & $-\odot .357781$ & 2.931324 \\
\hline $\mathrm{H}$ & 2.798183 & -0.472091 & 2.118488 \\
\hline $\mathrm{H}$ & 2.005858 & -1.921670 & 2.742983 \\
\hline $\mathrm{H}$ & -1.043865 & -1.468972 & 2.935053 \\
\hline $\mathrm{H}$ & -0.352493 & -3.060335 & 2.613721 \\
\hline $\mathrm{H}$ & -1.965142 & -2.668198 & 2.007434 \\
\hline
\end{tabular}

$\begin{array}{lrrr}\mathrm{C} & -3.704923 & -0.257949 & -1.015583 \\ \mathrm{C} & -0.161859 & 1.882988 & 0.064735 \\ \mathrm{C} & 0.445463 & 2.091629 & 1.329202 \\ \mathrm{C} & 1.381064 & 3.100380 & 1.514122 \\ \mathrm{C} & 1.728601 & 3.940638 & 0.449516 \\ \mathrm{C} & 1.147563 & 3.748947 & -0.809853 \\ \mathrm{C} & 0.232004 & 2.724487 & -1.006589 \\ \mathrm{H} & 1.536106 & -1.201028 & -2.341326 \\ \mathrm{H} & 3.995495 & -1.001523 & -2.500453 \\ \mathrm{H} & 5.398759 & -1.287926 & -0.468822 \\ \mathrm{H} & 4.335011 & -1.754809 & 1.727520 \\ \mathrm{H} & 1.883672 & -1.938473 & 1.903260 \\ \mathrm{H} & 0.180711 & -3.242561 & 2.341511 \\ \mathrm{H} & -1.028994 & -2.074989 & 2.898338 \\ \mathrm{H} & -1.552904 & -3.595091 & 2.186445 \\ \mathrm{H} & -0.936346 & -1.980742 & -2.130333 \\ \mathrm{H} & -2.590856 & -2.797049 & -0.448756 \\ \mathrm{H} & -0.494194 & 0.483118 & -2.198128 \\ \mathrm{H} & -3.667311 & -1.068575 & 1.025497 \\ \mathrm{H} & -2.000544 & 2.010903 & 2.213564 \\ \mathrm{H} & -2.206597 & 0.385955 & 2.907847 \\ \mathrm{H} & -3.555171 & 1.159311 & 2.103056 \\ \mathrm{H} & -2.859655 & 1.277446 & -2.337307 \\ \mathrm{H} & -2.737908 & -0.351311 & -2.987406 \\ \mathrm{H} & -4.310943 & -1.081872 & -1.407740 \\ \mathrm{H} & -4.400733 & 0.541956 & -0.743100 \\ \mathrm{H} & 0.212213 & 1.423212 & 2.150491 \\ \mathrm{H} & 1.847708 & 3.232623 & 2.485525 \\ \mathrm{H} & 2.452927 & 4.735795 & 0.598556 \\ \mathrm{H} & 1.412850 & 4.401604 & -1.635891 \\ \mathrm{H} & -0.220706 & 2.599851 & -1.984835\end{array}$

$E(U B+H F-L Y P)=-928.719674329$

$40 / 1$

RB3LYP/6-311+G(d,p) optimized structure:

$E(U B+H F-L Y P)=-928.673049607$

$41 / 2$

UB3LYP/6-311+G(d,p) optimized structure:

$\begin{array}{rr}C & 2.147605 \\ C & 3.528182 \\ C & 4.318208 \\ C & 3.718606 \\ C & 2.340057 \\ C & 1.522661 \\ C & 0.090373 \\ C & -0.837940 \\ C & -0.794754 \\ C & -0.979051 \\ C & -2.022138 \\ C & -1.231709 \\ C & -2.954293 \\ C & -2.092878 \\ C & -1.139513 \\ C & -2.464553 \\ C & -2.670669\end{array}$

$-1.451103$

$-1.542158$

$-0.397394$

0.841683

0.938185

$-0.211107$

$-0.160496$

$\odot .762726$

2.110197

$-1.204507$

$-0.151247$

$-1.490915$

$\odot .258104$

0.760779

$-0.151095$

2.057811

$-2.064145$

$\begin{array}{rr}2.646162 & -0.525494 \\ 3.900071 & 0.074492 \\ 4.680405 & 0.257151 \\ 4.190780 & -0.160387 \\ 2.936834 & -0.758730 \\ 2.142458 & -0.962798 \\ 0.829843 & -1.604318 \\ 0.172722 & -2.393869 \\ 0.483537 & -3.009374 \\ -0.310153 & -1.618646 \\ -1.068888 & -2.518516 \\ -1.145133 & -0.337994 \\ -2.391498 & -1.816284 \\ -2.113532 & -0.406978 \\ -1.450831 & 0.366347 \\ -2.647327 & -0.000029 \\ -2.484919 & -0.828541 \\ -3.240934 & -1.710184 \\ -1.039575 & 1.776833 \\ -0.379990 & 2.199384 \\ 0.007105 & 3.529040 \\ -0.249622 & 4.468938 \\ -0.891472 & 4.064374 \\ -1.275745 & 2.735158 \\ 2.047123 & -0.667657 \\ 4.268335 & 0.401574 \\ 5.657478 & 0.727208 \\ 4.785425 & -0.010457 \\ 2.557577 & -1.051039 \\ 1.559751 & -3.037921 \\ 0.007102 & -2.459819 \\ 0.102996 & -4.038032\end{array}$

$-1.456514$ $-1.552277$

$-0.409107$

0.831044

0.927695

$-0.217522$

$-0.153970$

0.733511

2. 059221

$-1.165307$

$-0.130253$

$-1.434975$

0.277978

0.770457

$-0.118668$

2.115982

$-2.057296$

$-1.023079$

0.077275

1.244670

1.412846

0.413801

$-0.758680$

$-0.925720$

$-2.351940$

$-2.521306$

$-0.481855$

1.728708

1.901858

2. 258186

2. 884288

2. 106751 


$\begin{array}{rrrr}\mathrm{H} & -0.084359 & -2.119604 & -2.116669 \\ \mathrm{H} & -1.287977 & -3.538805 & -0.477560 \\ \mathrm{H} & -0.621732 & 0.330475 & -2.125677 \\ \mathrm{H} & -2.928936 & -2.399482 & 1.035341 \\ \mathrm{H} & -2.567976 & 1.075674 & 2.291066 \\ \mathrm{H} & -2.119623 & -0.514428 & 2.932565 \\ \mathrm{H} & -3.704993 & -0.284535 & 2.207909 \\ \mathrm{H} & -3.092138 & 0.034576 & -2.350884 \\ \mathrm{H} & -2.274594 & -1.395625 & -2.973018 \\ \mathrm{H} & -3.421111 & -2.716103 & -1.422938 \\ \mathrm{H} & -4.220936 & -1.282295 & -0.785554 \\ \mathrm{H} & -0.143696 & 1.467860 & 2.011483 \\ \mathrm{H} & 0.522319 & 3.827745 & 2.322382 \\ \mathrm{H} & 0.054493 & 5.504261 & 0.543079 \\ \mathrm{H} & -1.094822 & 4.786480 & -1.545586 \\ \mathrm{H} & -1.781976 & 2.436604 & -1.840330\end{array}$

$E(R B+H F-L Y P)=-928.968168033$

\section{$50 / 1$}

RB3LYP/6-311+G(d,p) optimized structure:

\begin{tabular}{|c|c|c|c|}
\hline C & $\odot .411325$ & ๑. 616717 & 0.833734 \\
\hline C & -1.046810 & ๑. 093979 & 1.095057 \\
\hline C & -0.525467 & -1.328472 & 0.646073 \\
\hline C & ๑. 694271 & -0.643498 & -0.038060 \\
\hline C & -1.470606 & -2.131970 & -0.226083 \\
\hline 0 & -1.207341 & -2.639549 & -1.292076 \\
\hline C & -2.145624 & 0.509264 & 0.144538 \\
\hline 0 & -1.998943 & 0.700153 & -1.046220 \\
\hline C & -4.498078 & ๑. 212863 & -0.089705 \\
\hline C & -3.866427 & -2.242694 & -0.504598 \\
\hline 0 & -2.702882 & -2.135071 & $\odot .341240$ \\
\hline 0 & -3.356412 & ๑. 480792 & $\odot .755598$ \\
\hline C & -4.948250 & -1.266030 & $\odot .006900$ \\
\hline C & 2.043302 & -1.319776 & ๑. . 017797 \\
\hline C & 2.197365 & -2.615169 & -0.490514 \\
\hline C & 3.174649 & $-\odot .687532$ & 0.554442 \\
\hline C & 3.428316 & -3.273502 & -0.455597 \\
\hline C & 4.408999 & -1.327633 & 0.592602 \\
\hline C & 4.544075 & -2.628470 & ๑. .091632 \\
\hline 0 & 5.795023 & -3.174756 & ๑. 179991 \\
\hline C & 5.988313 & -4.485710 & -0.322633 \\
\hline C & -6.176467 & -1.423814 & -0.911846 \\
\hline C & -5.326238 & -1.610573 & 1.458813 \\
\hline C & ๑. 695490 & 2.020871 & 0.357231 \\
\hline C & 1.112610 & 2.984894 & 1.281783 \\
\hline C & 0.567487 & 2.421714 & -0.984490 \\
\hline C & 1.386925 & 4.303597 & $\odot .909830$ \\
\hline C & ๑. 840397 & 3.726231 & -1.373574 \\
\hline C & 1.250182 & 4.679559 & -0.430195 \\
\hline 0 & 1.491866 & 5.933110 & -0.916793 \\
\hline C & 1.906363 & 6.936001 & -0.004783 \\
\hline $\mathrm{H}$ & -1.404068 & 0.165778 & 2.123175 \\
\hline $\mathrm{H}$ & -0.230512 & -1.915409 & 1.522544 \\
\hline $\mathrm{H}$ & ๑ . 415170 & $-\odot .444655$ & -1.077817 \\
\hline $\mathrm{H}$ & -4.251959 & ๑. 491187 & -1.117144 \\
\hline H & -5.298327 & ๑. 862847 & ๑. 275581 \\
\hline $\mathrm{H}$ & -4.239373 & -3.272371 & -0.458609 \\
\hline $\mathrm{H}$ & -3.572880 & -2.028789 & -1.536746 \\
\hline $\mathrm{H}$ & 1.338904 & -3.117254 & -0.929899 \\
\hline $\mathrm{H}$ & 3.098655 & ๑. 326971 & $\odot .935720$ \\
\hline $\mathrm{H}$ & 3.504036 & -4.277495 & -0.858058 \\
\hline $\mathrm{H}$ & 5.284936 & $-\odot .836365$ & 1. $0 \odot 5207$ \\
\hline $\mathrm{H}$ & 5.772136 & -4.544016 & -1.397929 \\
\hline $\mathrm{H}$ & 7.041488 & -4.719512 & -0.154563 \\
\hline $\mathrm{H}$ & 5.366077 & -5.219284 & 0.207737 \\
\hline $\mathrm{H}$ & -6.987140 & $-\odot .757897$ & -0.594710 \\
\hline $\mathrm{H}$ & -5.936267 & -1.188972 & -1.955836 \\
\hline
\end{tabular}

$\begin{array}{rrrr}\mathrm{H} & -6.559742 & -2.449973 & -0.876375 \\ \mathrm{H} & -5.678270 & -2.646525 & 1.532916 \\ \mathrm{H} & -4.469666 & -1.490574 & 2.126159 \\ \mathrm{H} & -6.131939 & -0.956832 & 1.813650 \\ \mathrm{H} & 0.929351 & 0.477726 & 1.790804 \\ \mathrm{H} & 1.230605 & 2.704794 & 2.327004 \\ \mathrm{H} & 0.234393 & 1.710241 & -1.731080 \\ \mathrm{H} & 1.709163 & 5.013629 & 1.663101 \\ \mathrm{H} & 0.741704 & 4.033374 & -2.410194 \\ \mathrm{H} & 2.035128 & 7.844739 & -0.595905 \\ \mathrm{H} & 1.150937 & 7.113804 & 0.772522 \\ \mathrm{H} & 2.860347 & 6.678326 & 0.474708\end{array}$

$E(R B+H F-L Y P)=-1420.84195823$

$51 / 2$

UB3LYP/6-311+G(d,p) optimized structure:

\begin{tabular}{|c|c|c|c|}
\hline C & ๑.295973 & 0.717840 & 1. 001317 \\
\hline C & -0.948527 & -0.212246 & 1.244896 \\
\hline C & $-\odot .074190$ & -1.404516 & $\odot .706860$ \\
\hline C & $\odot .928715$ & $-\odot .367619$ & ๑.094367 \\
\hline C & -0.740543 & -2.339535 & -0.292208 \\
\hline 0 & -0.322718 & -2.572385 & -1.405043 \\
\hline C & -2.121111 & -0.001205 & 0.304811 \\
\hline 0 & -2.011856 & 0.475124 & -0.807551 \\
\hline C & -4.244360 & -1.016883 & -0.127153 \\
\hline C & -2.973202 & -3.175343 & -0.667436 \\
\hline 0 & -1.891742 & -2.794848 & 0.224488 \\
\hline 0 & -3.243756 & -0.534445 & $\odot .813129$ \\
\hline C & -4.289525 & -2.562548 & -0.145830 \\
\hline C & 2.393400 & $-\odot .675892$ & $\odot .138565$ \\
\hline C & 2.885459 & -1.746554 & -0.648657 \\
\hline C & 3.310934 & 0.048695 & $\odot .930568$ \\
\hline C & 4.224441 & -2.091498 & -0.644535 \\
\hline C & 4.653550 & -0.276360 & 0.942608 \\
\hline C & 5.127762 & -1.355584 & 0.158229 \\
\hline 0 & 6.440696 & -1.591735 & 0.241544 \\
\hline C & 7.032310 & -2.659743 & -0.513023 \\
\hline C & -5.400681 & -2.966135 & -1.137709 \\
\hline C & -4.610753 & -3.105519 & 1.258714 \\
\hline C & $\odot .105505$ & 2.108833 & 0.474319 \\
\hline C & -0.768093 & $3.0 \odot 2953$ & 1.137879 \\
\hline C & 0.814904 & 2.581626 & -0.653330 \\
\hline C & -0.932133 & 4.307696 & $\odot .713393$ \\
\hline C & 0.669237 & 3.880393 & -1.092853 \\
\hline C & -0.210228 & 4.762039 & -0.417210 \\
\hline 0 & $-\odot .287327$ & 5.994476 & -0.923299 \\
\hline C & -1.159304 & 6.970743 & -0.332650 \\
\hline $\mathrm{H}$ & -1.289603 & -0.313234 & 2.275318 \\
\hline $\mathrm{H}$ & 0.356731 & -1.971911 & 1.537230 \\
\hline $\mathrm{H}$ & $\odot .613551$ & -0.215021 & -0.944374 \\
\hline $\mathrm{H}$ & -4.026671 & $-\odot .598845$ & -1.112768 \\
\hline $\mathrm{H}$ & -5.200484 & -0.622542 & ๑.225747 \\
\hline $\mathrm{H}$ & -3.037209 & -4.267612 & -0.671177 \\
\hline $\mathrm{H}$ & -2.725414 & -2.836518 & -1.677310 \\
\hline $\mathrm{H}$ & 2.184676 & -2.303033 & -1.265189 \\
\hline $\mathrm{H}$ & 2.969757 & 0.886317 & 1.530472 \\
\hline $\mathrm{H}$ & 4.570827 & -2.915423 & -1.257038 \\
\hline $\mathrm{H}$ & 5.369628 & 0.278067 & 1.539844 \\
\hline $\mathrm{H}$ & 6.893397 & -2.497218 & -1.586748 \\
\hline $\mathrm{H}$ & 8.093176 & -2.631966 & -0.267906 \\
\hline $\mathrm{H}$ & 6.606439 & -3.623829 & -0.216792 \\
\hline $\mathrm{H}$ & -6.370226 & -2.571787 & -0.814611 \\
\hline $\mathrm{H}$ & -5.202935 & -2.591491 & -2.148996 \\
\hline $\mathrm{H}$ & -5.491897 & -4.056308 & -1.193273 \\
\hline $\mathrm{H}$ & -4.695078 & -4.197983 & 1.239675 \\
\hline $\mathrm{H}$ & -3.833737 & -2.835133 & 1.977942 \\
\hline $\mathrm{H}$ & -5.564766 & -2.704347 & 1.619271 \\
\hline
\end{tabular}




$\begin{array}{rr}\mathrm{H} & 0.831390 \\ \mathrm{H} & -1.322342 \\ \mathrm{H} & 1.487245 \\ \mathrm{H} & -1.606750 \\ \mathrm{H} & 1.207457 \\ \mathrm{H} & -1.030427 \\ \mathrm{H} & -2.200479 \\ \mathrm{H} & -0.869864\end{array}$

\begin{abstract}
0.762900
2. 658962

1.916373

4.969310

4.254849

7.870756

6.636362

7.168993
\end{abstract}

$\begin{array}{ll}H & -2.410945 \\ H & -6.035379 \\ H & -5.428352 \\ H & -6.418158\end{array}$

$-5.520105$

$-6.215749$

$-4.759366$

$-4.593879$

$-1.065062$

1. 287023

2. 128951

$\odot .637984$

$E(U B+H F-L Y P)=-1420.60158807$

$60 / 1$

RB3LYP/6-311+G(d,p) optimized structure:

$61 / 2$

UB3LYP/6-311+G(d,p) optimized structure:

\begin{tabular}{|c|c|c|c|}
\hline C & -1.267083 & -1.079261 & -1.344285 \\
\hline C & -1.195732 & ๑. 284961 & -1.259034 \\
\hline C & 1.940284 & 1.848048 & 0.451774 \\
\hline C & 2.204767 & ๑. 632775 & ○. 997872 \\
\hline C & ๑. 812933 & 2.658685 & ๑. 967287 \\
\hline 0 & 0.036228 & 2.291685 & 1.829930 \\
\hline C & -2.076626 & 1.271360 & -0.589067 \\
\hline 0 & -3.111621 & 1.061545 & 0.017526 \\
\hline C & -2.281605 & 3.596375 & -0.148721 \\
\hline C & -0.232258 & 4.802481 & 0.766623 \\
\hline 0 & 0.783275 & 3.857317 & 0.352248 \\
\hline 0 & -1.557760 & 2.501893 & -0.764130 \\
\hline C & -1.434845 & 4.875696 & -0.196566 \\
\hline C & 3.287795 & $-\odot .262539$ & 0.667743 \\
\hline C & 4.267073 & $\odot .023935$ & -0.317902 \\
\hline C & 3.398569 & -1.490941 & 1.371138 \\
\hline C & 5.298465 & -0.854030 & -0.590677 \\
\hline C & 4.419044 & -2.378405 & 1. 111161 \\
\hline C & 5.386789 & -2.070303 & ๑. 125512 \\
\hline 0 & 6.340410 & -2.989894 & -0.051653 \\
\hline C & 7.384864 & -2.769270 & -1.009668 \\
\hline C & -2.335885 & 6.019491 & 0.318811 \\
\hline C & -0.969424 & 5.179215 & -1.631515 \\
\hline C & -2.163632 & -2.095404 & -0.846884 \\
\hline C & -3.317966 & -1.872215 & -0.042795 \\
\hline C & -1.856778 & -3.443281 & -1.203846 \\
\hline C & -4.111107 & -2.921089 & 0.376437 \\
\hline C & -2.638345 & -4.494867 & -0.793718 \\
\hline C & -3.784389 & -4.246814 & 0.006605 \\
\hline 0 & -4.481612 & -5.326868 & 0.353654 \\
\hline C & -5.667709 & -5.199078 & 1.155790 \\
\hline $\mathrm{H}$ & -0.368320 & 0.749692 & -1.785992 \\
\hline $\mathrm{H}$ & 2.542452 & 2.292164 & -0.334235 \\
\hline $\mathrm{H}$ & 1.530424 & ๑. 309238 & 1.789354 \\
\hline $\mathrm{H}$ & -2.531884 & 3.312653 & ๑. 876695 \\
\hline $\mathrm{H}$ & -3.215435 & 3.732520 & -0.705411 \\
\hline $\mathrm{H}$ & ๑. 283023 & 5.766468 & 0.779253 \\
\hline $\mathrm{H}$ & -0.553867 & 4.548070 & 1.779257 \\
\hline $\mathrm{H}$ & 4.217051 & ๑. 955975 & -0.871443 \\
\hline $\mathrm{H}$ & 2.663522 & -1.727961 & 2.135238 \\
\hline $\mathrm{H}$ & 6.033127 & -0.602617 & -1.346176 \\
\hline $\mathrm{H}$ & 4.512798 & -3.315700 & 1.649080 \\
\hline $\mathrm{H}$ & 7.961151 & -1.873593 & -0.755697 \\
\hline $\mathrm{H}$ & 8.023652 & -3.649409 & -0.947456 \\
\hline $\mathrm{H}$ & 6.972473 & -2.680642 & -2.020276 \\
\hline $\mathrm{H}$ & -3.212333 & 6.141040 & -0.326519 \\
\hline $\mathrm{H}$ & -2.691056 & 5.832232 & 1.339223 \\
\hline $\mathrm{H}$ & -1.792054 & 6.970197 & 0.320283 \\
\hline $\mathrm{H}$ & -0.426464 & 6.130463 & -1.666129 \\
\hline $\mathrm{H}$ & -0.309337 & 4.396296 & -2.012519 \\
\hline $\mathrm{H}$ & -1.829106 & 5.261125 & -2.306479 \\
\hline $\mathrm{H}$ & -0.454853 & -1.503761 & -1.933614 \\
\hline $\mathrm{H}$ & -3.570255 & -0.856533 & 0.234463 \\
\hline $\mathrm{H}$ & -0.983213 & -3.639826 & -1.819194 \\
\hline $\mathrm{H}$ & -4.983205 & -2.717576 & ๑. 986348 \\
\hline
\end{tabular}

\begin{tabular}{|c|c|c|}
\hline-1.580522 & $-\odot .931860$ & $-\odot .988472$ \\
\hline-1.090033 & 0.331137 & -0.910334 \\
\hline 2.630900 & 1.444802 & $\odot .413988$ \\
\hline 2.671927 & 0.260610 & 1.056139 \\
\hline 1.793598 & 2.540683 & 0.932313 \\
\hline 1.128303 & 2.510552 & 1.951465 \\
\hline-1.685360 & 1.565624 & -0.376147 \\
\hline-2.777146 & 1.732513 & 0.143130 \\
\hline-1.230226 & 3.865455 & $-\odot .024098$ \\
\hline 1.105027 & 4.783316 & $\odot .467604$ \\
\hline 1.873988 & 3.622351 & $\odot .109145$ \\
\hline-0.807422 & 2.593007 & -0.544414 \\
\hline$-\odot .189004$ & 4.946297 & $-\odot .357298$ \\
\hline 3.436858 & -0.929388 & 0.698949 \\
\hline 4.338317 & $-\odot .979307$ & $-\odot .378515$ \\
\hline 3.278019 & -2.101439 & 1.467483 \\
\hline 5.048225 & -2.136122 & -0.685147 \\
\hline 3.975344 & -3.262604 & 1.174826 \\
\hline 4.868799 & -3.290235 & 0.093249 \\
\hline 5.509593 & -4.472119 & -0.116410 \\
\hline 6.433040 & -4.561994 & -1.192051 \\
\hline-0.805036 & 6.298590 & 0.060281 \\
\hline 0.126838 & 4.955001 & -1.862794 \\
\hline-2.822786 & -1.605677 & $-\odot .620618$ \\
\hline-3.942087 & -1.009721 & $-\odot .0 \odot 7995$ \\
\hline-2.902833 & -2.989040 & $-\odot .909347$ \\
\hline-5.079695 & -1.752316 & ๑. 298017 \\
\hline-4.027640 & -3.736940 & $-\odot .609738$ \\
\hline-5.132160 & -3.120900 & $-\odot .000305$ \\
\hline-6.193970 & -3.932068 & 0.257517 \\
\hline-7.342988 & -3.367243 & 0.873044 \\
\hline-0.084905 & 0.481210 & -1.291482 \\
\hline 3.188450 & 1.647688 & -0.494795 \\
\hline 2.055407 & 0.178738 & 1.950539 \\
\hline-1.373663 & 3.773785 & 1.057476 \\
\hline-2.197720 & 4.120476 & $-\odot .471091$ \\
\hline 1.766851 & 5.632782 & 0.269014 \\
\hline$\odot .879351$ & 4.743405 & 1.536337 \\
\hline 4.496724 & -0.096106 & -0.990453 \\
\hline 2.588955 & -2.091624 & 2.308373 \\
\hline 5.735762 & -2.131121 & -1.523044 \\
\hline 3.849532 & -4.162899 & 1.767765 \\
\hline 7.264124 & -3.855662 & -1.068817 \\
\hline 6.819673 & -5.582162 & -1.167898 \\
\hline 5.944449 & -4.381746 & -2.158334 \\
\hline-1.717464 & 6.503174 & -0.510986 \\
\hline-1.064988 & 6.314136 & 1.125910 \\
\hline-0.104656 & 7.120740 & -0.126727 \\
\hline ๑.811366 & 5.776047 & -2.108215 \\
\hline$\odot .592416$ & 4.016325 & -2.171001 \\
\hline$-\odot .788554$ & 5.093149 & -2.450647 \\
\hline$-\odot .871607$ & -1.626131 & -1.441629 \\
\hline-3.910574 & 0.045848 & 0.228978 \\
\hline-2.055197 & -3.480803 & -1.381326 \\
\hline-5.918864 & -1.253828 & 0.769939 \\
\hline-4.080345 & -4.797433 & -0.834903 \\
\hline-8.056161 & -4.186399 & 0.980434 \\
\hline-7.108422 & -2.957158 & 1.863701 \\
\hline-7.785825 & -2.578687 & ๑. 25121 \\
\hline
\end{tabular}


$E(R B+H F-L Y P)=-1420.85060294$

7 0/1

RB3LYP/6-311+G(d,p) optimized structure:

$\begin{array}{rrrr}\text { F } & 3.747475 & 0.103396 & -1.090559 \\ \text { C } & 2.958038 & -0.000070 & 0.000319 \\ \text { C } & 2.020804 & 1.249428 & 0.114965 \\ \text { C } & 0.616052 & -0.730938 & -0.005199 \\ \text { C } & 0.616070 & 0.730891 & 0.005133 \\ \text { C } & 2.020779 & -1.249474 & -0.115151 \\ \text { F } & 3.746352 & -0.103634 & 1.092007 \\ \text { F } & 2.342870 & 2.139220 & -0.870053 \\ \text { F } & 2.260306 & 1.882061 & 1.306884 \\ \text { C } & -1.844615 & 0.410712 & -1.949553 \\ \text { C } & -1.810325 & -0.647603 & 0.416762 \\ \text { C } & -0.537910 & -1.456172 & 0.096722 \\ \text { C } & -1.844359 & -0.410682 & 1.949686 \\ \text { F } & 2.260374 & -1.881418 & -1.307421 \\ \text { F } & 2.342738 & -2.139840 & 0.869373 \\ \text { C } & -0.803023 & -2.879760 & 0.000967 \\ \text { S } & -3.238100 & 1.789242 & -0.007575 \\ \text { S } & -3.238188 & -1.789121 & 0.007900 \\ \text { C } & -0.537866 & 1.456161 & -0.096794 \\ \text { C } & 0.227715 & 3.974858 & 0.085968 \\ \text { C } & 0.227500 & -3.974879 & -0.086689 \\ \text { C } & -2.136087 & 3.144811 & 0.052568 \\ \text { H } & -2.557757 & 4.140551 & 0.139550 \\ \text { C } & -1.810355 & 0.647639 & -0.416633 \\ \text { C } & -2.136242 & -3.144721 & -0.052659 \\ \text { C } & -0.802892 & 2.879782 & -0.001235 \\ \text { H } & -2.557967 & -4.140427 & -0.139762 \\ \text { H } & 0.832743 & 4.032585 & -0.823712 \\ \text { H } & 0.918416 & 3.804674 & 0.916935 \\ \text { H } & -0.260377 & 4.941967 & 0.237627 \\ \text { H } & 0.918143 & -3.804460 & -0.917658 \\ \text { H } & -0.260675 & -4.941899 & -0.238647 \\ \text { H } & 0.832598 & -4.032971 & 0.822920 \\ \text { H } & -2.759421 & 0.102208 & 2.251891 \\ \text { H } & -0.985000 & 0.189599 & 2.262897 \\ \text { H } & -1.797739 & -1.375086 & 2.461857 \\ \text { H } & -0.985324 & -0.189604 & -2.262881 \\ \text { H } & -1.798028 & 1.375112 & -2.461734 \\ \text { H } & -2.759742 & -0.102141 & -2.251625\end{array}$

$E(R B+H F-L Y P)=-2051.61025405$

$71 / 2$

UB3LYP/6-311+G(d,p) optimized structure:

$\begin{array}{lr}\text { F } & 4.089700 \\ C & 2.945494 \\ C & 2.056303 \\ C & 0.638141 \\ C & 0.635377 \\ C & 2.055910 \\ \text { F } & 3.207915 \\ F & 2.385786 \\ F & 2.259455 \\ C & -1.906764 \\ \text { C } & -1.780877 \\ C & -0.540680 \\ C & -1.733354 \\ F & 2.327201 \\ F & 2.312823 \\ C & -0.796156 \\ \text { S } & -3.230468\end{array}$

0.017852
-0.012531
1.237703
-0.712579
0.707201
-1.231645
-0.074870
1.792712
2.167942
0.315175
-0.648972
-1.455144
-0.351333
-1.617837
-2.276087
-2.835738
1.775039

$-0.403804$

0.289404

0.018019

0.029059

0.000437

$-0.088171$

1.613108

$-1.178854$

0.983091

$-1.867729$

0.540547

0.142935

2.065520

$-1.366992$

$\odot .732654$

$-0.070935$

$\odot .041526$

\begin{tabular}{|c|c|c|c|}
\hline $\mathrm{s}$ & -3.222288 & -1.795332 & $\odot .267212$ \\
\hline C & -0.549664 & 1.442140 & $-\odot .093733$ \\
\hline C & 0.220057 & 3.955902 & -0.018968 \\
\hline C & $\odot .201640$ & -3.917423 & -0.386503 \\
\hline C & -2.151761 & 3.107954 & ๑. 055031 \\
\hline H & -2.569209 & 4.107190 & 0.130353 \\
\hline C & -1.819980 & $\odot .621895$ & -0.342324 \\
\hline C & -2.157865 & -3.103898 & -0.030916 \\
\hline C & $-\odot .792487$ & 2.842110 & -0.031083 \\
\hline H & -2.585094 & -4.089456 & $-\odot .187804$ \\
\hline H & 0.979077 & 3.810828 & -0.791349 \\
\hline H & 0.738239 & 4.010579 & 0.942820 \\
\hline $\mathrm{H}$ & $-\odot .273021$ & 4.914948 & -0.194829 \\
\hline $\mathrm{H}$ & $\odot .894966$ & -3.601408 & -1.170292 \\
\hline $\mathrm{H}$ & -0.311276 & -4.820506 & -0.725269 \\
\hline $\mathrm{H}$ & 0.799595 & -4.171719 & 0.493438 \\
\hline H & -2.634807 & $\odot .172299$ & 2.391431 \\
\hline $\mathrm{H}$ & -0.862061 & $\odot .264281$ & 2.304938 \\
\hline $\mathrm{H}$ & -1.656518 & -1.286053 & 2.626162 \\
\hline $\mathrm{H}$ & -1.046527 & -0.280651 & -2.184681 \\
\hline $\mathrm{H}$ & -1.902165 & 1.248708 & -2.434897 \\
\hline $\mathrm{H}$ & -2.822839 & $-\odot .228951$ & -2.106846 \\
\hline
\end{tabular}

$E(U B+H F-L Y P)=-2051.36998356$

$81 / 2$

UB3LYP/6-311+G(d,p) optimized structure:

\begin{tabular}{|c|c|c|c|}
\hline $\mathrm{F}$ & 4.162111 & $-\odot .160507$ & -0.463737 \\
\hline C & 2.978363 & 0.011035 & $\odot .133869$ \\
\hline C & 2.108164 & 1.100928 & $-\odot .539930$ \\
\hline C & $\odot .666670$ & $-\odot .668192$ & 0.106047 \\
\hline C & ๑.681401 & $\odot .642411$ & -0.268100 \\
\hline C & 2.073784 & -1.244717 & ๑. 059747 \\
\hline $\mathrm{F}$ & 3.159618 & ๑. 341887 & 1.434770 \\
\hline $\mathrm{F}$ & 2.333587 & 1.109252 & -1.880799 \\
\hline $\mathrm{F}$ & 2.396063 & 2.332150 & -0.061816 \\
\hline C & -1.465825 & -0.236376 & -2.344829 \\
\hline C & -1.454696 & $-\odot .902309$ & 1.443145 \\
\hline C & $-\odot .506359$ & -1.429400 & $\odot .524081$ \\
\hline C & -1.379573 & 0.326234 & 2.288579 \\
\hline $\mathrm{F}$ & 2.310403 & -1.870207 & -1.130406 \\
\hline $\mathrm{F}$ & 2.313933 & -2.123908 & 1.055232 \\
\hline C & $-\odot .833458$ & -2.756793 & ๑. 092858 \\
\hline S & -2.814112 & 2.087374 & -1.592395 \\
\hline S & -2.766169 & -2.010018 & 1.693020 \\
\hline C & -0.505127 & 1.446400 & -0.561732 \\
\hline C & ๑.012840 & 3.552465 & 0.928530 \\
\hline C & $-\odot .026276$ & -3.633147 & $-\odot .825242$ \\
\hline C & $-2.049 \odot 46$ & 3.173295 & $-\odot .47949 \odot$ \\
\hline H & -2.547985 & 4.092700 & -0.197387 \\
\hline C & -1.496302 & $\odot .966401$ & -1.458046 \\
\hline C & -2.043987 & -3.163583 & 0.619070 \\
\hline C & $-\odot .814846$ & 2.738200 & $-\odot .029188$ \\
\hline H & -2.547188 & -4.102401 & 0.421715 \\
\hline H & -0.620916 & 4.250264 & 1.482223 \\
\hline H & 0.767140 & 4.137345 & ๑.393289 \\
\hline H & ๑.546931 & 2.925047 & 1.647061 \\
\hline H & $\odot .911458$ & -3.937036 & -0.349873 \\
\hline H & $\odot .235900$ & -3.121874 & -1.755971 \\
\hline H & -0.585523 & -4.537649 & -1.075722 \\
\hline $\mathrm{H}$ & -1.718802 & 0.113971 & 3.307548 \\
\hline H & -2.019592 & 1.128647 & 1.895335 \\
\hline H & $-\odot .356513$ & 0.704374 & 2.339486 \\
\hline H & -2.091536 & -1.046602 & -1.945515 \\
\hline H & -0.447026 & -0.614249 & -2.455316 \\
\hline H & 50812 & ๑.0०6988 & -3.340312 \\
\hline
\end{tabular}

$E(U B+H F-L Y P)=-2051.35657196$ 
8 0/1

RB3LYP/6-311+G(d,p) optimized structure:

$\begin{array}{lrrr}\text { F } & 4.125802 & -0.156970 & -0.404432 \\ \mathrm{C} & 2.930784 & 0.093493 & 0.163477 \\ \mathrm{C} & 2.040695 & 1.034041 & -0.672825 \\ \mathrm{C} & 0.630859 & -0.651960 & 0.180300 \\ \mathrm{C} & 0.628012 & 0.600652 & -0.339508 \\ \mathrm{C} & 2.050866 & -1.168903 & 0.313993 \\ \mathrm{~F} & 3.131359 & 0.647123 & 1.387796 \\ \mathrm{~F} & 2.296393 & 0.840734 & -2.003116 \\ \mathrm{~F} & 2.307457 & 2.338715 & -0.405098 \\ \mathrm{C} & -1.417560 & -0.164526 & -2.543600 \\ \mathrm{C} & -1.336587 & -1.037411 & 1.675708 \\ \mathrm{C} & -0.518250 & -1.456091 & 0.645532 \\ \mathrm{C} & -1.258711 & 0.210729 & 2.504422 \\ \mathrm{~F} & 2.387326 & -2.051746 & -0.678075 \\ \mathrm{~F} & 2.265758 & -1.794369 & 1.498051 \\ \mathrm{C} & -0.892967 & -2.753184 & 0.110844 \\ \mathrm{~S} & -2.670200 & 2.262644 & -1.875103 \\ \mathrm{~S} & -2.584003 & -2.207645 & 1.990575 \\ \mathrm{C} & -0.529974 & 1.442517 & -0.702384 \\ \mathrm{C} & -0.045700 & 3.414006 & 0.976752 \\ \mathrm{C} & -0.182540 & -3.491806 & -0.993905 \\ \mathrm{C} & -1.987118 & 3.256007 & -0.630535 \\ \mathrm{H} & -2.461013 & 4.190763 & -0.361072 \\ \mathrm{C} & -1.422364 & 1.067019 & -1.686038 \\ \mathrm{C} & -1.999276 & -3.251905 & 0.738414 \\ \mathrm{C} & -0.846178 & 2.723394 & -0.098641 \\ \mathrm{H} & -2.500546 & -4.188610 & 0.533245 \\ \mathrm{H} & -0.677162 & 4.108014 & 1.540035 \\ \mathrm{H} & 0.785312 & 3.985227 & 0.551350 \\ \mathrm{H} & 0.388804 & 2.701664 & 1.685098 \\ \mathrm{H} & 0.778322 & -3.893823 & -0.657880 \\ \mathrm{H} & 0.027388 & -2.843681 & -1.851219 \\ \mathrm{H} & -0.794077 & -4.328668 & -1.344303 \\ \mathrm{H} & -1.645735 & 0.040727 & 3.514117 \\ \mathrm{H} & -1.834571 & 1.029978 & 2.057432 \\ \mathrm{H} & -0.220196 & 0.542914 & 2.595892 \\ & -1.968159 & -0.987091 & -2.070869 \\ \mathrm{H} & -1.874900 & -0.028054 & -3.519195\end{array}$

$E(R B+H F-L Y P)=-2051.63219952$

\section{0/1}

RB3LYP/6-311+G(d,p) optimized structure:

$\begin{array}{lr}\mathrm{H} & -1.829247 \\ \mathrm{C} & -1.815034 \\ \mathrm{C} & -1.815235 \\ \mathrm{C} & -0.663098 \\ \mathrm{C} & -3.048970 \\ \mathrm{C} & -3.049070 \\ \mathrm{C} & -0.663187 \\ \mathrm{C} & 0.857845 \\ \mathrm{H} & -6.432036 \\ \mathrm{C} & -4.298182 \\ \mathrm{H} & 1.213146 \\ \mathrm{H} & -1.829608 \\ \mathrm{H} & 1.213404 \\ \mathrm{C} & 1.614355 \\ \mathrm{C} & 1.614353 \\ \mathrm{C} & 0.857725 \\ \mathrm{C} & 3.102130 \\ \mathrm{C} & 2.889779 \\ \mathrm{C} & 1.094814\end{array}$

$-1.637528$

$-0.933681$

0.933558

$-0.462697$

$-0.464400$

0.464174

0.462630

$-0.568624$

$-0.802435$

0.900126

1.514764

1.637458

$-1.514546$

$-0.266635$

0.568775

0.352190

$-0.813578$

$\odot .593388$ $\odot .266817$

$\begin{array}{lrrr}\mathrm{C} & 1.831560 & -3.968837 & 0.900354 \\ \mathrm{C} & 3.629266 & -3.190238 & -0.507257 \\ \mathrm{H} & 3.304951 & -1.299413 & -1.485347 \\ \mathrm{H} & 0.102296 & -2.691426 & 1.015913 \\ \mathrm{H} & 1.410142 & -4.717768 & 1.566128 \\ \mathrm{H} & 4.615201 & -3.328155 & -0.943797 \\ \mathrm{H} & 3.674508 & -5.048700 & 0.588973 \\ \mathrm{C} & 3.102470 & 4.155662 & -0.352089 \\ \mathrm{C} & 1.093993 & 2.826011 & -0.591365 \\ \mathrm{C} & 2.890794 & 2.046453 & 0.811904 \\ \mathrm{C} & 3.630443 & 3.189000 & 0.505533 \\ \mathrm{C} & 1.830915 & 3.970077 & -0.898365 \\ \mathrm{H} & 0.100729 & 2.693759 & -1.012437 \\ \mathrm{H} & 3.306601 & 1.297407 & 1.482271 \\ \mathrm{H} & 4.617166 & 3.325911 & 0.940608 \\ \mathrm{H} & 1.408844 & 4.719922 & -1.562696 \\ \mathrm{H} & 3.674987 & 5.048551 & -0.588898 \\ \mathrm{H} & -4.296530 & 1.903958 & 1.599920 \\ \mathrm{C} & -5.488734 & 0.541334 & 0.455085 \\ \mathrm{C} & -4.297990 & -1.071540 & -0.900421 \\ \mathrm{C} & -5.488637 & -0.541753 & -0.455448 \\ \mathrm{H} & -4.296191 & -1.904228 & -1.600209 \\ \mathrm{H} & -6.432207 & 0.954169 & 0.802021\end{array}$

$E(R B+H F-L Y P)=-925.380586862$

$91 / 2$

UB3LYP/6-311+G(d,p) optimized structure:

\begin{tabular}{|c|c|c|c|}
\hline $\mathrm{H}$ & -1.800789 & -2.040135 & -1.500020 \\
\hline C & -1.791914 & -1.165763 & -0.854635 \\
\hline C & -1.791783 & 1.165797 & 0.854840 \\
\hline C & $-\odot .627473$ & -0.552322 & -0.428887 \\
\hline C & -3.022117 & -0.581929 & -0.426241 \\
\hline c & -3.022051 & 0.582005 & $\odot .426578$ \\
\hline C & $-\odot .627409$ & $\odot .552315$ & 0.428969 \\
\hline C & 0.880566 & -0.610041 & -0.576116 \\
\hline $\mathrm{H}$ & -6.409150 & -1.003722 & -0.734656 \\
\hline C & -4.263717 & 1.130442 & ๑.825133 \\
\hline $\mathrm{H}$ & 1.213635 & 0.159486 & 1.515982 \\
\hline $\mathrm{H}$ & -1.800559 & 2.040171 & 1.500224 \\
\hline $\mathrm{H}$ & 1.213463 & -0.159548 & -1.516100 \\
\hline C & 1.622548 & -1.869293 & -0.292765 \\
\hline C & 1.622632 & 1.869222 & $\odot .292604$ \\
\hline C & ๑.880648 & $\odot .609988$ & 0.576034 \\
\hline C & 3.052629 & -4.223057 & 0.246033 \\
\hline C & 2.825438 & -2.147132 & -0.970940 \\
\hline C & 1.152839 & -2.791101 & $\odot .666593$ \\
\hline C & 1.860221 & -3.958295 & $\odot .930519$ \\
\hline C & 3.533338 & -3.313451 & -0.705325 \\
\hline $\mathrm{H}$ & 3.196072 & -1.443496 & -1.711947 \\
\hline $\mathrm{H}$ & 0.230736 & -2.585549 & 1.204399 \\
\hline $\mathrm{H}$ & 1.490034 & -4.662333 & 1.669612 \\
\hline $\mathrm{H}$ & 4.456713 & -3.521020 & -1.237427 \\
\hline $\mathrm{H}$ & 3.607670 & -5.132711 & $\odot .455046$ \\
\hline C & 3.052710 & 4.222953 & -0.246343 \\
\hline C & 1.152855 & 2.791029 & -0.666722 \\
\hline C & 2.825587 & 2.147044 & 0.970669 \\
\hline C & 3.533485 & 3.313347 & 0.704980 \\
\hline C & 1.860237 & 3.958207 & -0.930722 \\
\hline $\mathrm{H}$ & 0.230701 & 2.585490 & -1.204444 \\
\hline $\mathrm{H}$ & 3.196273 & 1.443408 & 1.711651 \\
\hline $\mathrm{H}$ & 4.456912 & 3.520904 & 1.237000 \\
\hline $\mathrm{H}$ & 1.489998 & 4.662244 & -1.669790 \\
\hline $\mathrm{H}$ & 3.607750 & 5.132595 & -0.455414 \\
\hline $\mathrm{H}$ & -4.268489 & 2.006347 & 1.468185 \\
\hline C & -5.470062 & 0.565761 & 0.412381 \\
\hline C & -4.263843 & -1.130324 & -0.824663 \\
\hline C & -5.470125 & $-\odot .565602$ & -0.411781 \\
\hline
\end{tabular}


$\begin{array}{rrrr}H & -4.268714 & -2.006229 & -1.467714 \\ H & -6.409038 & 1.003912 & 0.735356\end{array}$

$E(U B+H F-L Y P)=-925.118726654$

\section{9-10 TS $1 / 2$}

UB3LYP/6-311+G(d,p) optimized structure:

\begin{tabular}{|c|c|c|c|}
\hline C & -1.531534 & -0.451459 & 1.415587 \\
\hline C & -1.008192 & -0.180365 & 2.715615 \\
\hline C & $-0.69379 \odot$ & $-\odot .246588$ & 0.340641 \\
\hline C & $\odot .344135$ & ๑. 298124 & 2.869195 \\
\hline C & 1.164348 & $\odot .515905$ & 1.721481 \\
\hline C & 0.606564 & ๑.261070 & $\odot .487277$ \\
\hline C & -0.704399 & -0.490333 & -1.134385 \\
\hline $\mathrm{H}$ & -0.316001 & -1.466386 & -1.428045 \\
\hline C & $\odot .970812$ & $\odot .440593$ & $-\odot .951561$ \\
\hline H & $\odot .668805$ & 1.400776 & -1.371817 \\
\hline C & 2.151135 & -0.137993 & -1.572739 \\
\hline C & 2.843793 & -1.218003 & $-\odot .973844$ \\
\hline C & 3.973802 & -1.749053 & -1.579371 \\
\hline C & 4.426890 & -1.225297 & -2.798422 \\
\hline C & 3.745040 & -0.164443 & -3.411854 \\
\hline C & 2.616562 & 0.371441 & -2.809547 \\
\hline $\mathrm{H}$ & 4.504751 & -2.572802 & -1.112400 \\
\hline $\mathrm{H}$ & 5.308393 & -1.646409 & -3.272743 \\
\hline $\mathrm{H}$ & 4.102769 & 0.238402 & -4.354285 \\
\hline H & 2.087347 & 1.197678 & -3.277153 \\
\hline C & -1.699261 & 0.054220 & -2.044026 \\
\hline C & -2.509537 & 1.155305 & -1.675330 \\
\hline C & -3.459629 & 1.653289 & -2.555632 \\
\hline C & -3.611122 & 1.074627 & -3.823661 \\
\hline C & -2.807764 & $-\odot .0 \odot 80 \odot 5$ & -4.209872 \\
\hline C & -1.858413 & -0.510970 & -3.332850 \\
\hline $\mathrm{H}$ & -4.082643 & 2.493540 & -2.265179 \\
\hline $\mathrm{H}$ & -4.351799 & 1.469824 & -4.512559 \\
\hline $\mathrm{H}$ & -2.932097 & -0.453325 & -5.192044 \\
\hline $\mathrm{H}$ & -1.236941 & -1.353742 & -3.624225 \\
\hline $\mathrm{H}$ & -2.383989 & 1.606068 & $-\odot .695 \odot 26$ \\
\hline $\mathrm{H}$ & 2.485319 & -1.626361 & -0.033267 \\
\hline $\mathrm{H}$ & -2.537816 & -0.847605 & 1.308368 \\
\hline $\mathrm{H}$ & 2.169225 & $\odot .912110$ & 1.840957 \\
\hline C & $\odot .832673$ & 0.550310 & 4.174686 \\
\hline C & 0.034214 & $\odot .344405$ & 5.288253 \\
\hline $\mathrm{H}$ & 1.849959 & 0.913540 & 4.292996 \\
\hline $\mathrm{H}$ & 0.426517 & 0.546361 & 6.280092 \\
\hline C & -1.795785 & $-\odot .378845$ & 3.876146 \\
\hline C & -1.285462 & -0.121136 & 5.138343 \\
\hline $\mathrm{H}$ & -2.813733 & -0.742009 & 3.763458 \\
\hline $\mathrm{H}$ & -1.904437 & $-\odot .282353$ & 6.015448 \\
\hline
\end{tabular}

$E(U B+H F-L Y P)=-925.116651167$

$101 / 2$

UB3LYP/6-311+G(d,p) optimized structure:

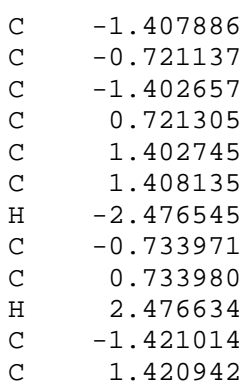

0.106579 0.045331 0.080560 $-0.045382$ $-0.080583$ $-0.106656$ 0.228381

0.057435 $-0.057432$ $-0.228404$ 0.307702 $-0.307670$
$\mathrm{H}$

C

C

C

C

$\mathrm{H}$

H

$\mathrm{H}$

C

C

C

C

$\mathrm{H}$

H

H

H

$\mathrm{H}$

$\mathrm{C}$

$\mathrm{H}$
0.134663

$-0.725958$

○. 726010

$-0.134626$

0.118603

0.752176

$-0.853303$

$-0.739716$

0.870694

1. 382245

$-1.517205$

$-1.309366$

1.563672

○. 218263

$-0.118563$

$-0.752178$

0.853379

$\odot .739793$

$-0.870694$

$-1.382275$

1. 517309

1.309475

$-1.563701$

$-0.218221$

○. 055248

$-0.185979$

0.185903

$-0.055350$

$\odot .096150$

$-\odot .096272$

$E(U B+H F-L Y P)=-925.150060755$

10 0/1

RB3LYP/6-311+G(d,p) optimized structure:

\begin{tabular}{|c|c|c|c|}
\hline $\mathrm{C}$ & -1.408731 & 3.760409 & -0.110379 \\
\hline $\mathrm{C}$ & -0.727441 & 2.490981 & -0.066817 \\
\hline C & -1.408896 & 1.292697 & -0.115422 \\
\hline $\mathrm{C}$ & ๑. 727661 & 2.490908 & ๑. 066802 \\
\hline C & 1.408992 & 1.292555 & ๑. 115420 \\
\hline $\mathrm{C}$ & 1.409080 & 3.760267 & 0.110367 \\
\hline $\mathrm{H}$ & -2.492916 & 1.315052 & -0.126753 \\
\hline $\mathrm{C}$ & -0.742426 & ๑. 030256 & 0.013215 \\
\hline $\mathrm{C}$ & ๑. 742391 & 0.030185 & -0.013213 \\
\hline $\mathrm{H}$ & 2.493016 & 1.314790 & ๑. 126773 \\
\hline C & -1.411569 & -1.150690 & ๑. 302819 \\
\hline $\mathrm{C}$ & 1.411432 & -1.150825 & -0.302781 \\
\hline C & -2.821630 & -1.477051 & $\odot .162237$ \\
\hline $\mathrm{H}$ & ๑. 809596 & -1.942945 & -0.742540 \\
\hline $\mathrm{H}$ & $-\odot .809797$ & -1.942825 & $\odot .742638$ \\
\hline $\mathrm{C}$ & 2.821481 & -1.477250 & -0.162220 \\
\hline C & 5.541176 & -2.230365 & ๑. . 040087 \\
\hline C & 3.671614 & -0.894288 & 0.804662 \\
\hline C & 3.372003 & -2.476387 & - ๑. 996894 \\
\hline C & 4.710751 & -2.841076 & $-\odot .904072$ \\
\hline C & 5.010530 & -1.262610 & ๑. 897697 \\
\hline $\mathrm{H}$ & 3.263633 & -0.178358 & 1.510665 \\
\hline $\mathrm{H}$ & 2.731109 & -2.955127 & -1.733553 \\
\hline $\mathrm{H}$ & 5.108886 & -3.604961 & -1.566861 \\
\hline $\mathrm{H}$ & 5.641087 & -0.803704 & 1.654904 \\
\hline $\mathrm{H}$ & 6.586404 & -2.516802 & $\odot .117390$ \\
\hline C & -5.541348 & -2.230094 & -0.040099 \\
\hline C & -3.671693 & -0.894208 & $-\odot .804782$ \\
\hline C & -3.372234 & -2.476054 & ๑. 997019 \\
\hline C & -4.710992 & -2.840703 & $\odot .904187$ \\
\hline C & -5.010619 & -1.262491 & - ๑. 897828 \\
\hline $\mathrm{H}$ & -3.263648 & -0.178422 & -1.510892 \\
\hline $\mathrm{H}$ & -2.731394 & -2.954717 & 1.733775 \\
\hline
\end{tabular}

2.491351

09567

4.938406

5.884917

5.884835

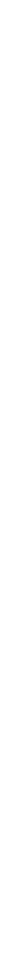

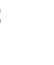




$\begin{array}{rrrr}\text { H } & -5.109188 & -3.604477 & 1.567067 \\ H & -5.641116 & -0.803681 & -1.655143 \\ \text { H } & -6.586583 & -2.516502 & -0.117414 \\ \text { C } & -0.717570 & 4.932432 & -0.054188 \\ \text { H } & 2.493447 & 3.757769 & 0.193182 \\ H & -2.493098 & 3.758021 & -0.193191 \\ \text { C } & 0.718038 & 4.932360 & 0.054178 \\ H & -1.245072 & 5.881637 & -0.093138 \\ \text { H } & 1.245635 & 5.881512 & 0.093131 \\ & & & \\ \text { E(RB+HF-LYP })= & -925.353575744 & \end{array}$

